\title{
Dynamic Revenue Maximization with Heterogeneous Objects: A Mechanism Design Approach
}

\author{
Alex Gershkov and Benny Moldovanu*
}

26.2.2008

\begin{abstract}
We study the revenue maximizing allocation of several heterogeneous, commonly ranked objects to impatient agents with privately known characteristics who arrive sequentially according to a Poisson process. There is a deadline after which no more objects can be allocated. We first characterize implementable allocation schemes, and compute the expected revenue for any implementable, deterministic and Markovian allocation policy. The revenue-maximizing policy is obtained by a variational argument which sheds more light on its properties than the usual dynamic programming approach. In particular, we show that this policy does not depend on the characteristics of the available objects at each point in time. Finally, we use our main result in order to: a) establish a comparison with the welfare maximizing policy; b) derive the optimal inventory choice; c) explain empirical regularities about pricing in clearance sales.
\end{abstract}

\section{Introduction}

We study the following dynamic mechanism design problem in continuous time: a designer has to allocate (or assign) a finite set of heterogeneous objects with known characteristics to a stream of randomly arriving, impatient agents with privately known characteristics. There is a deadline by which all objects must be sold ${ }^{1}$. The objects are substitutes, and each agent derives utility from at most one object. Moreover, all agents rank the available objects in the same way, and values for objects have a multiplicative structure involving the agents' and objects' types. Under the assumption that monetary transfers are feasible, we characterize the dynamic revenue-maximizing policy and the associated dynamic pricing scheme. We also show how important features of the revenue maximizing policy can be used to yield a straightforward solution to the larger optimization problem where the designer also chooses the size and quality composition of its inventory.

Dynamic pricing and assignment problems appear in numerous frameworks such as the retail of seasonal and style goods, the allocation of fixed capacities in the travel and leisure industries (e.g., airlines, trains, hotels, rental cars), the allocation of a fixed inventory of

${ }^{*}$ This manuscript supplants the previously circulated discussion paper "The Dynamic Assignment of Heterogeneous Objects: A Mechanism Design Approach" . A coeditor and four anonymous referees made many helpful comments. We are grateful for financial support from the German Science Foundation, and from the Max Planck Society. We wish to thank Heidrun Hoppe, Paul Klemperer, and participants at the Conference on "Computational Social Systems", Dagstuhl 2007, and at seminars in Oxford, Warwick and Bruxelles for helpful remarks. Gershkov, Moldovanu: Department of Economics, University of Bonn, Lennestr. 37,53113 Bonn.mold@uni-bonn.de, alex.gershkov@uni-bonn.de

${ }^{1}$ Simpler techniques can be applied to the setting where the horizon is infinite and there is time discounting. All our main results are easily extended to this situation 
equipment in a given period of time (e.g., equipment for medical procedures), the assignment of personnel to incoming tasks. More recently, dynamic pricing methods play an increased role in the allocation of electricity and bandwidth.

The main trade-off in our paper is as follows: assigning an object today means that the valuable option of assigning it in the future - possibly to an agent who values it more-, is foregone; on the other hand, since the arrival process of agents is stochastic, and since there is a deadline, the "future" may never materialize. The revenue maximizing policy needs to define prices for each available objects such that, at each point in time, this trade-off is optimally taken into account.

Our paper provides three main contributions:

1. By allowing for heterogeneous objects, we broaden the scope of large literatures in Economics and Management Science that analyze pricing decisions under stochastic demand conditions (in the MS literature this is called revenue or yield management). This broader perspective allows us to integrate in a seamless way quantity/quality optimization with the traditional price considerations.

2. We introduce to the above literature a new technical method which has two components: a) A focus - inspired by the "mechanism design philosophy" and the payoff/revenue equivalence principle - on implementable allocation policies rather than on prices; b) A variational method that yields more insight than the traditional dynamic programming/Bellman's equation method. Our approach yields new results even for the much studied case of dynamic revenue-maximization for identical goods. Moreover, it facilitates the calculation and assessment of the revenue associated to simple policies that may be used in practice (even if they are not optimal in some sense) and reveals properties that are common to other interesting allocation policies (e.g., dynamic welfare maximization).

3. Our analysis yields many testable implications about the pattern of prices in relevant situations exhibiting fixed inventories of substitute goods that need to be sold by a deadline. An example concerning clearance sales for apparel is provided here.

Whereas the large literature on yield or revenue management has directly focused on revenue-maximizing pricing (mostly for the special case of our model where agents have linear, private values for identical objects) our approach starts by a characterization of all dynamically implementable, non-randomized allocation policies. Such policies are described by partitions of the set of possible agent types: an arriving agent gets the best available object if his type lies in the highest interval of the partition, the second best available object if his type lies in the second highest interval, and so on... These intervals generally depend on the point in time of the arrival, and on the composition of the set of available objects at that point in time. For implementable allocation policies we derive the associated menus of prices (one menu for each point in time, and for each subset of remaining objects) that implement it, and show that these menus have an appealing recursive structure: each agent who is assigned an object has to pay the value he displaces in terms of the chosen allocation.

We next turn to revenue-maximization. Using several basic results about the Poisson stochastic process, we first compute the revenue generated by any individual-rational, nonrandomized, Markovian and implementable allocation policy. Then, we can directly use variational arguments in order to characterize the revenue-maximizing allocation policy. The associated optimal prices are of "secondary importance" since they are completely determined by the implementation conditions. 
Whereas the optimal prices necessarily depend on the composition of inventory, our main result is that, at each point in time, the revenue maximizing allocation policy depends only on the size of the available inventory, but not on its exact composition. To understand the meaning of this somewhat surprising result, consider the same model, but with identical objects. Then, for each size of available inventory, and for each point in time, the revenue maximizing allocation policy is characterized by a single cut-off type: only an arriving agent with type above that cut-off obtains one of the objects. In contrast, when objects are heterogeneous, the revenue maximizing policy is, at each point in time, and for each subset of available objects, characterized by several cut-off types which determine if the arriving agent gets the best available object, the second best, etc... Our result says that for any subset of $k$ available heterogeneous objects, and for any point in time, the highest cut-off coincides with the optimal cut-off in a situation with one available object, the second-highest cut-off coincides with the optimal cutoff in a situation with two identical objects, and so on till the lowest cut-off which coincides with the optimal cut-off in a situation with an inventory of $k$ identical objects.

The last part of the paper is devoted to several applications, all heavily using the characterization result described above:

1. We perform a comparison of the welfare maximizing and revenue maximizing allocation policies. The dynamic welfare-maximizing allocation policy has been characterized for the complete information case - via a system of differential equations - by Albright [1]. Since that policy is non-randomized, Markovian and has the form of a partition, it can be implemented also in our private information framework by the dynamic price schedules identified above. The resulting scheme coincides then with a dynamic version of the Vickrey-Clarke-Groves mechanism. We show that, for distributions of agents' types that have an increasing failure (or hazard) rate, the revenue maximizing policy is, overall, strictly more conservative: at any point in time, the cutoff curves defining the revenue maximizing dynamic policy are strictly above the respective cutoff curves defining the welfare maximizing policy ${ }^{2}$.

2. We embed revenue maximization in the larger optimization problem where, before sales begin, the seller chooses the size and composition of the inventory . We show how the formulae we derived for revenue maximization (together with information about marginal costs) immediately yield a set of intuitive equations that characterize the optimal number of objects and their qualities.

3. We derive some testable implications about the pattern of observed prices for different qualities in clearance sales, and confront them with available data. Compared to standard models that only consider identical objects, our analysis offers a somewhat more convincing explanation for several well-known observed regularities. For example, we explain why the average clearance mark-down (in percentage terms) is higher for the higher quality, more expensive product lines.

The rest of the paper is organized is follows: In the remainder of this Section we review the related literature.

In Section 2 we present the continuous-time model of sequential assignment of heterogeneous objects to randomly arriving, privately informed agents. Section 3 focuses on a

\footnotetext{
${ }^{2}$ We also show that this result may fail if the hazard rate condition is not fulfilled, even if virtual valuations are increasing.
} 
characterization of implementable policies, and of the associated menus of dynamic prices that implement such policies.

In Section 4 we study the revenue maximizing policy. We obtain a general expression for expected revenue given any Markovian, non-randomized allocation policy, and we use a variational argument in order to derive functional equations that characterize the revenue maximizing allocation policy and the expected revenue generated by this policy.

In Section 5 we exhibit three different applications of our main result: In Subsection 5.1 we show that the revenue maximizing policy is, at each point in time, more conservative than the dynamically efficient policy if the distribution of values has an increasing hazard (or failure) rate. In Subsection 5.2 we embed our revenue maximization in a larger maximization problem where the designer also chooses the size and composition of the inventory. In Subsection 5.3 we derive several empirically testable implications and show how these can be used to explain observed phenomena.

Section 6 concludes. Most of the proofs are relegated to an Appendix.

\section{$1.1 \quad$ Related Literature}

Derman, Lieberman and Ross [9] introduced an elegant discrete-time model where a set of distinct, commonly ranked objects needs to be assigned to a set of sequentially arriving agents that have a multiplicative utility function involving their type and the object's properties. They characterize the dynamic, welfare-maximizing allocation under a complete information assumption about the agents' valuations ${ }^{3}$. Albright [1] extended the Derman-LiebermanRoss model and characterization to the continuous-time framework with random arrivals of agents - this framework is used here. Incomplete information in a similar, but static model with non-random demand is introduced in the classic paper by Mussa and Rosen [24] who focused on a monopolist's price/quality decisions.

There are very large theoretical and applied literatures on dynamic pricing of inventories (sometimes called revenue or yield management) both in Economics and in the fields of Management and Operations Research. The general goal in this literature is revenue maximization. We refer the reader to the excellent literature surveys and discussions of involved issues by Bitran and Caldentey [7] and Elmaghraby and Keskinocak [11], and to the comprehensive book by Talluri and Van Ryzin [35]. McAfee and te Velde [21] survey the applications to the airline industry who has pioneered many of the modern practices in revenue management.

Riley and Zeckhauser [32] considered a single object revenue-maximizing procedure where there is learning about the distribution of the agents' values. In their model, the optimal mechanism is a sequence of take-it-or-leave-it offers. Lazear [18] offers a theory of clearance sales that allows for several identical units. In his model all customers have the same value, and the seller learns about this value over time. It is shown that a pattern of decreasing prices is optimal. Pashigian [28], Pashigian and Bowen [29], and Pashigian, Bowen and Gould [30] test some of the empirical implications of Lazear's analysis based on data obtained from several industries

In a continuous-time framework with stochastic arrivals of agents, Kincaid and Darling [17], and Gallego and Van Ryzin [12] use dynamic programming in order to characterize - implicitly via Bellman's equations - the revenue maximizing pricing policy for a set of identical objects that need to be sold before a deadline. A main result is that the expected

\footnotetext{
${ }^{3}$ Similar analytical methods - involving dynamic programming and optimal stopping - have been fruitfully used in the large literature on search- see for example the surveys of Lippman and McCall [19] and Mortensen $[23]$.
} 
revenue in the optimal policy - which is characterized for each size of inventory by a single posted price - is increasing and concave both in the number of objects and in the length of time left till the deadline. Moreover, each relevant cutoff price drops with time as long as there is no sale, but jumps up after each sale ${ }^{4}$. These authors were able to calculate in closed form the solution for what amounts (in our terms) to an exponential distribution of agents' values. McAfee and te Velde [20] find an explicit solution for a Pareto distributions of agents' values, and show that it coincides with the welfare maximizing policy. Generally, a closed form solution is not available, and even the expression of expected revenue as a function of the optimal cutoff prices is not available in the literature.

Arnold and Lippman [2], Das Varma and Vettas [10] and Gallien [14] consider the same basic problem as above, but in a framework with an infinite horizon and discounting. In this case, the revenue maximizing posted prices - again one price for each size of inventory -, turn out to be stationary: they do not depend on time ${ }^{5}$. This stationarity allows Gallien to offer a reasonable sufficient condition ensuring that all sales occur immediately upon arrival, even if the arriving agents can strategically delay their purchase. Also in an infinite horizon model, Wang [36] compares a posted-price regime with an auction one. In his framework, the auction option is costly.

Lazear [18] argues that delayed purchases by strategic consumers is not likely to be significant if the number of consumers is large, but a more recent, still small, strand of the literature focuses on strategic buyer behavior in models with fixed inventories ${ }^{6}$. Nocke and Peitz [26] exhibit conditions for a decreasing price path to be optimal even if consumers are strategic about the timing of purchases in a model with identical units and fixed demand. These authors do not consider pricing policies that condition on past realized sales. Aviv and Pazgal [4] assume that prices must be declining (which is not innocuous) in a twoperiod model with stochastic arrivals, but derive analytic results only for a policies that do not depend on past realized sales. Su [34] allows more general policies, but assumes a deterministic demand flow.

To conclude, as Bitran and Caldentey [7] noted, due to the technical complexity, the literature on dynamic revenue maximization with stochastic demand has focused on models with identical objects, contrasting the present framework. ${ }^{7}$ Moreover, no solution is yet available to the Gallego\&van Ryzin [12] type of models with strategic customers.

\section{The Model}

There are $n$ items (or objects) that need to be allocated to arriving agents before a deadline $T>0$. Each item $i$ is characterized by a "quality" $q_{i}$. Agents (or buyers) arrive according to a Poisson process with intensity $\lambda$, and each can only be served upon arrival (i.e., agents are impatient). After an item is assigned, it cannot be reallocated in the future. Each agent

\footnotetext{
${ }^{4}$ Bitran and Mondschein [6] obtain similar results in a discrete time framework.

${ }^{5}$ Das Varma and Vettas consider a model with discrete time and deterministic arrivals, whereas Gallien and Arnold and Lippman have continuous time models with stochastic arrivals. The latter authors assume (rather than derive) the stationarity of posted prices, and also compare these to reservation prices in a model where arriving agents announce bids.

${ }^{6}$ This should be contrasted with the earlier literature on the so-called "Coase Conjecture" where the inventory can be replenished. McAfee and Wiseman [22] show that the introduction of capacity costs counters the Coasian insight. For other papers that examine the relationship between limited inventories and pricing schemes see Harris and Raviv [16] and Wilson [37].

${ }^{7}$ See Gallego and Van Ryzin [13] for an exception. Some models assume that customers belong to several known classes which allows the use of third-degree price discrimination.
} 
$j$ is characterized by a "type" $x_{j}$. An agent with type $x_{j}$ who obtains an item with quality $q_{i}$ enjoys a utility of $q_{i} x_{j}$. The designer (or seller) seeks to maximize expected revenue.

While the items' types $0 \leq q_{n} \leq q_{n-1} \leq \ldots \leq q_{1}$ are assumed to be known constants, the agents' types are assumed to be represented by independent and identically distributed random variables $X_{i}$ on $[0,+\infty)$ with common twice differentiable c.d.f. $F$ ( $f$ denotes the corresponding density function). The realization of $X_{i}$ is private information to agent $i$. We assume that each $X_{i}$ has a finite mean, denoted by $\mu$, and a finite variance. Moreover, we assume that $f(x)<\infty$ and that $x-\frac{1-F(x)}{f(x)}$ increases for any $x \in[0, \infty){ }^{8}$

The multiplicative structure employed above is restrictive: it is one of the simplest structures allowing a meaningful treatment of several qualities while allowing the modeling of private information as a one-dimensional variable. The present formulation is standard in the relevant literature, following the seminal static analysis in Mussa and Rosen [24].

Another restrictive assumption pertains to the fact that agents are infinitely impatient. In particular, strategic considerations about the moment of purchase do not play a role in our present analysis ${ }^{9}$. Alternatively, we could assume that agents bear a high enough cost of waiting per unit of time.

\section{Illustration of the Main Result}

Consider a situation with two objects having qualities $q_{1}>q_{2}$. The seller's problem is to find a pricing scheme that maximizes expected revenue. For each instance $t$, a pricing scheme $P_{t}=\left\{P_{t}^{1}\left(\left\{q_{1}, q_{2}\right\}\right), P_{t}^{2}\left(\left\{q_{1}, q_{2}\right\}\right), P_{t}^{1}\left(\left\{q_{1}\right\}\right), P_{t}^{2}\left(\left\{q_{2}\right\}\right)\right\}$ needs to specify the price charged for object $i$ given any possible inventory available at that time. Let us first analyze the optimal choice of the arriving agents:

1. If at time $t$ both objects are available, an agent with type $x$ buys the object with quality $q_{1}$ if and only if $q_{1} x-P_{t}^{1}\left(\left\{q_{1}, q_{2}\right\}\right) \geq \max \left\{q_{2} x-P_{t}^{2}\left(\left\{q_{1}, q_{2}\right\}\right), 0\right\}$, and buys the object with quality $q_{2}$ if and only if $q_{2} x-P_{t}^{2}\left(\left\{q_{1}, q_{2}\right\}\right) \geq \max \left\{q_{1} x-P_{t}^{1}\left(\left\{q_{1}, q_{2}\right\}\right), 0\right\}$. If $\max \left\{q_{2} x-P_{t}^{2}\left(\left\{q_{1}, q_{2}\right\}\right), q_{1} x-P_{t}^{1}\left(\left\{q_{1}, q_{2}\right\}\right)\right\}<0$, the arriving agent buys nothing.

2. If at time $t$ only one object of quality $q_{i}, i=1,2$ is available, the arriving agent buys it if and only if $q_{i} x-P_{t}^{i}\left(\left\{q_{i}\right\}\right) \geq 0$.

Given the above considerations, the seller's problem can be written as

$$
\begin{aligned}
& \max _{P_{t}} \int_{0}^{T}\left[P_{t}^{1}\left(\left\{q_{1}, q_{2}\right\}+\int_{t}^{T} P_{s}^{2}\left(\left\{q_{2}\right\}\right) h_{2}(s) d s\right] h_{1,\left\{q_{1}, q_{2}\right\}}(t) d t\right. \\
& +\int_{0}^{T}\left[P_{t}^{2}\left(\left\{q_{1}, q_{2}\right\}+\int_{t}^{T} P_{s}^{1}\left(\left\{q_{1}\right\}\right) h_{1}(s) d s\right] h_{2,\left\{q_{1}, q_{2}\right\}}(t) d t\right.
\end{aligned}
$$

where $h_{i,\left\{q_{1}, q_{2}\right\}}(t), i=1,2$ is the density of a first arrival that leads to a sale of the object with quality $q_{i}$ at time $t$ (given that both objects are still available), and $h_{i}(s)$ is the density that at time $s$ the second object of quality $q_{i}$ will be sold (given that the other object was

\footnotetext{
${ }^{8}$ The expression $x-\frac{1-F(x)}{f(x)}$ is the so-called virtual valuation of the buyer of type $x$. For a detailed discussion see Myerson [25] and Bulow and Roberts [8].

${ }^{9}$ This is not a serious constraint in the discounted infinite horizon model because of the stationarity of the optimal policies there- see also Gallien [14]
} 
sold beforehand). It is important to note that these densities also depend on the pricing policy $P_{t}$ !

Assuming that buyers' values are exponentially distributed, and that buyers arrive according to a Poisson process with parameter $\lambda=1$, the solution of the above problem is given by the following four pricing curves:

$$
\begin{aligned}
P_{t}^{1}\left(\left\{q_{1}, q_{2}\right\}\right) & =\left(q_{1}-q_{2}\right) \ln (e+T-t)+q_{2} \ln \left(\frac{e^{2}}{2(e+T-t)}+\frac{1}{2}(e+T-t)\right) \\
P_{t}^{2}\left(\left\{q_{1}, q_{2}\right\}\right. & =q_{2} \ln \left(\frac{e^{2}}{2(e+T-t)}+\frac{1}{2}(e+T-t)\right) \\
P_{t}^{i}\left(\left\{q_{i}\right\}\right) & =q_{i} \ln (e+T-t), i=1,2
\end{aligned}
$$

A seminal result of the mechanism design theory - the so called "Payoff and Revenue Equivalence Theorem" - implies that, instead of focusing on pricing policies, we can choose instead an optimal allocation policy. An inspection of the above solution reveals a remarkable feature of the underlying allocation policy:

When both objects are still available, the agent arriving at time $t$ gets the best object with quality $q_{1}$ if and only if his type $x$ exceeds $\ln (e+T-t)$; if his type is in the interval $\left[\ln \left(\frac{e^{2}}{2(e+T-t)}+\frac{1}{2}(e+T-t)\right), \ln (e+T-t)\right)$ then he gets the with quality $q_{2}$; lower types get nothing. If only one object is available, the agent arriving at $t$ gets that object if and only if his type exceeds $\ln (e+T-t)$. In other words we have found that the revenue maximizing allocation policy is, in fact, characterized only by two curves such that:

1. The allocation policy is determined by cutoffs that are independent of the qualities of the available objects.

2. If only one object is available, then the respective allocation policy coincides with the policy governing the allocation of the best out of two available objects ${ }^{10}$.

In the general case with $n$ different objects the optimal pricing scheme needs to specify $\sum_{k=1}^{n} k\left(\begin{array}{l}n \\ k\end{array}\right)=n 2^{n-1}$ pricing curves, which, a-priori, makes the problem hardly tractable. Using a variational argument on the revenue maximizing policy, we show below that properties 1,2 above are not due to a coincidence: the optimal allocation policy - which completely determines the pricing policy via the payoff equivalence theorem - is always characterized by only $n$ curves.

\section{Implementable Policies}

Without loss of generality, we restrict attention to direct mechanisms where every agent, upon arrival, reports his characteristic $x_{i}$ and where the mechanism specifies an allocation (which item, if any, the agent gets) and a payment. The schemes we develop also have an obvious and immediate interpretation as indirect mechanisms, where the designer sets a time-dependent menu of prices, one for each item, and where arriving agents choose out that menu.

\footnotetext{
${ }^{10}$ If the two objects are identical, i.e., $q_{1}=q_{2}$, the above described cutoffs are still optimal, but at each $t$ instance only one of them is of relevance: this feature makes then the problem amenable to an analysis via dynamic programming and Bellman's equation.
} 
An allocation policy is called non-randomized and Markovian if, at any time $t$, and for any possible type of agent arriving at $t$, it uses a non-random allocation rule that only depends on the arrival time $t$, on the declared type of the arriving agent, and on the set of items available at $t$, denoted by $\Pi_{t}$. Thus, the policy depends on past decisions only via the state variable $\Pi_{t}$. We also restrict attention to interim-individually rational policies, where no agent ever pays more than the utility obtained from the physical allocation.

Denote by $Q_{t}:[0,+\infty) \times \Pi_{t} \rightarrow \Pi_{t} \cup \emptyset$ a non-randomized Markovian allocation policy for time $t$ and by $P_{t}:[0,+\infty) \times \Pi_{t} \rightarrow \mathbb{R}$ the associated payment rule. Denote also by $k_{t}$ the cardinality of set $\Pi_{t}$.

The next Proposition shows that a non-randomized, Markovian allocation policy is implementable if and only if it based on a partition of the agents' type space. ${ }^{11}$ In other words, implementability reduces here to setting a menu of prices, one for which object, from which the arriving agent has to choose.

Proposition 1 Assume that $\Pi_{t}$ is the set of objects available at time $t$, and assume that $q_{j} \neq q_{k}$ for any $q_{j}, q_{k} \in \Pi_{t}, j \neq k$.

1. A non-randomized, Markovian policy $Q_{t}$ is implementable if and only if there exist $k_{t}+1$ functions $\infty=y_{0, \Pi_{t}}(t) \geq y_{1, \Pi_{t}}(t) \geq y_{2, \Pi_{t}}(t) \geq \cdots \geq y_{k_{t}, \Pi_{t}}(t) \geq 0$, such that $x \in\left[y_{j, \Pi_{t}}(t), y_{j-1, \Pi_{t}}(t)\right) \Rightarrow Q_{t}\left(x, \Pi_{t}\right)=q_{(j)}$ where $q_{(j)}$ denotes the $j$ 'th highest element of the set $\Pi_{t}$, and such that $x<y_{k_{t}, \Pi_{t}}(t) \Rightarrow Q_{t}\left(x, \Pi_{t}\right)=\emptyset .{ }^{12}$

2. The associated payment scheme is given by $P_{t}\left(x, \Pi_{t}\right)=\sum_{i=j}^{k_{t}}\left(q_{(i)}-q_{(i+1)}\right) y_{i, \Pi_{t}}(t)+$ $S(t)$ if $x \in\left[y_{j, \Pi_{t}}(t), y_{j-1, \Pi_{t}}(t)\right)$ where $S(t)$ is some allocation- and type-independent function.

Proof. $\Longrightarrow$ If two reports of the agent that arrives at $t$ lead to the same physical allocation, then, in any incentive compatible mechanism, the associated payments should be the same as well. Denote by $P_{j}$ the payment that will be charged for the object with quality $q_{j}$. A direct mechanism is equivalent to a mechanism where the agent arriving at time $t$ chooses an object and a payment from a menu $\left(q_{j}, P_{j}\right)_{j=1}^{k_{t}}$. If some type $x$ prefers the pair $\left(q_{k}, P_{k}\right)$ over any other pair $\left(q_{l}, P_{l}\right)$ with $q_{k}>q_{l}$, then any type $\widetilde{x}>x$ also prefers $\left(q_{k}, P_{k}\right)$ over $\left(q_{l}, P_{l}\right)$. This implies that $Q_{t}\left(\widetilde{x}, \Pi_{t}\right) \geq Q_{t}\left(x, \Pi_{t}\right)$ for any $t$ and $\Pi_{t}$. Finally, noting that $Q_{t}\left(x, \Pi_{t}\right)=\emptyset$ is equivalent to allocating an object with quality equal to zero, implies that an agent who arrives at time $t$ gets object $q_{(k)}$ if he reports a type contained in the interval $\left(y_{k, \Pi_{t}}(t), y_{k-1, \Pi_{t}}(t)\right)$. A similar argument shows that $Q_{t}\left(y_{i, \Pi_{t}}(t), \Pi_{t}\right) \in\left\{q_{(i+1)}, q_{(i)}\right\}$ for $i \in\left\{1,2, \ldots, k_{t}\right\} .{ }^{13}$

$\Longleftarrow$ The proof is constructive: given a partition-based policy, we design a payment scheme $P_{t}\left(x, \Pi_{t}\right)$ that, for any $j \in\left\{1, \ldots, k_{t}\right\}$, induces type $x \in\left[y_{j, \Pi_{t}}(t), y_{j-1, \Pi_{t}}(t)\right)$ to choose the object with type $q_{(j)}$. Without loss of generality, we assume that an agent whose type is on the boundary between two intervals in the partition chooses the item with higher type. Consider then the following payment scheme

$$
P_{t}\left(x, \Pi_{t}\right)=\sum_{i=j}^{k_{t}}\left(q_{(i)}-q_{(i+1)}\right) y_{i, \Pi_{t}}(t)+S(t) \text { if } x \in\left[y_{j, \Pi_{t}}(t), y_{j-1, \Pi_{t}}(t)\right)
$$

\footnotetext{
${ }^{11}$ The result holds for any deterministic policy. But, since the rest of the analysis focuses on the Markov case, and in order to save on notational complexity, we consider only this case here.

${ }^{12}$ Types at the boundary between two intervals can be assigned to either one of the neighboring elements of the partition. That is, if $x_{i} \in\left\{y_{k_{t}, \Pi_{t}}(t), y_{k_{t}-1, \Pi_{t}}(t), \ldots, y_{2, \Pi_{t}}(t), y_{1, \Pi_{t}}(t)\right\}$, then $Q_{t}\left(y_{i, \Pi_{t}}(t), \Pi_{t}\right) \in$ $\left\{q_{i}, q_{i+1}\right\}, i=1,2, . ., k_{t}$.

${ }^{13}$ We thank a referee for simplifying our previous argument.
} 
where $S(t)$ is is some allocation- and type-independent function. Note that type $x=y_{j, \Pi_{t}}(t)$ is indifferent between $\left(q_{(j)}, P_{j}\right)$ and $\left(q_{(j+1)}, P_{j+1}\right)$. Moreover, any type above $y_{j, \Pi_{t}}(t)$ prefers $\left(q_{(j)}, P_{j}\right)$ over $\left(q_{(j+1)}, P_{j+1}\right)$, while any type below prefers $\left(q_{(j+1)}, P_{j+1}\right)$ over $\left(q_{(j)}, P_{j}\right)$. Therefore, any type $x \in\left[y_{j, \Pi_{t}}(t), y_{j-1, \Pi_{t}}(t)\right)$ prefers $\left(q_{(j)}, P_{j}\right)$ over any other pairs in the menu ${ }^{14}$.

The payment scheme has an intuitive interpretation: assume for a moment that the analyzed setup is the static one with $k_{t}$ objects and $k_{t}+1$ agents where, in addition to an agent with type $x$, there are $k_{t}$ other "dummy" agents with types $y_{1, \Pi_{t}}(t), y_{2, \Pi_{t}}(t), \cdots, y_{k_{t}, \Pi_{t}}(t)$. The payment for the object with the $j$-highest quality, $\sum_{i=j}^{k_{t}}\left(q_{(i)}-q_{(i+1)}\right) y_{i, \Pi_{t}}(t)$, represents the externality imposed by the agent with type $x_{i}$ on the dummy agents in the corresponding efficient allocation.

\section{Dynamic Revenue Maximization}

In this section we solve the dynamic revenue maximization problem. A main feature that differentiates our analysis from previous ones is the fact that we use the mechanism design approach developed in Section 4, and the insight behind the celebrated payoff/revenue equivalence theorem. Thus, we focus on the dynamic allocation policy that underlies revenue maximization, while pricing plays only a "secondary" role since, once the allocation is fixed, it is automatically induced by the implementation requirements.

Without loss of generality, we can restrict attention to Markovian, non-randomized poli$\operatorname{cies}^{15}$. The optimality of Markovian, possibly randomized, policies is standard for all models where, as is the case here, the instantaneous rewards and transition probabilities are historyindependent - see for example Theorem 11.1.1 in Puterman, 2005 which shows that, for any history-dependent policy, there is a Markovian, possibly randomized, policy with the same payoff. Given a Markovian policy, at each period $t$ the designer's problem is equivalent to a static problem where one object out of a given inventory needs to be allocated to a privately informed agent, and where the seller has different salvage values for the remaining possible inventories (the salvage values correspond to the various continuation values in the dynamic case). Analogously to Myerson (1981), the static revenue-maximization problem has a nonrandomized solution as long as the agent's virtual valuation is increasing (as assumed here): if at all, the agent should get the object for which virtual valuation plus salvage value is highest. Thus, at each period $t$ in the dynamic problem the seller has a non-randomized optimal policy as well.

We first calculate the expected revenue for any given Markovian, non-randomized allocation policy, and then we use a variational argument to derive the cut-off curves describing the revenue-maximizing dynamic policy.

Recall from Proposition 1 that, in order to implement a Markovian, non-randomized allocation which is given by $\infty=y_{0, \Pi_{t}}(t) \geq y_{1, \Pi_{t}}(t) \geq y_{2, \Pi_{t}}(t) \geq \cdots \geq y_{k_{t}, \Pi_{t}}(t)>0, \forall t$, the price at period $t$ for the object with the $j$-th highest characteristic (among the remaining

\footnotetext{
${ }^{14}$ If there are some identical objects, there exist implementable policies that do not take the form of partitions. But, for each such policy, there exists another implementable policy that is based on a partition, and that generates the same expected utility for all agents and for the designer.

${ }^{15}$ Theorem 2.7 in Gihman and Skorohod [15] gives sufficient conditions ensuring that jump Markovian controlled processes have optimal controls that are non-randomized and Markovian. Note though, that here we have also to respect an implementability (i.e., monotonicity) constraint.
} 
ones) needs to be

$$
P_{t}^{(j)}\left(\Pi_{t}\right)=\sum_{i=j}^{k_{t}}\left(q_{(i)}-q_{(i+1)}\right) y_{i, \Pi_{t}}(t)+S(t)
$$

if he gets the item with the $j^{\prime}$ th highest quality. In any interim individually rational mechanism we must have $S(t) \leq 0$, and, in order to maximize the revenue, we must clearly have $S(t)=0$.

After using simple properties of sampling out of Poisson processes (see the Proof of Proposition 7 in the Appendix), the expected revenue at time $t$ where $\Pi_{t} \neq \emptyset$ takes the form

$$
R\left(\Pi_{t}, t\right)=\sum_{i=1}^{k_{t}} \int_{t}^{T}\left(P_{s}^{(i)}\left(\Pi_{t}\right)+R\left(\Pi_{t} \backslash\left\{q_{(i)}\right\}, s\right)\right) h_{i, \Pi_{t}}(s) d s
$$

where

$$
h_{i, \Pi_{t}}(s)=\lambda\left[F\left(y_{i-1, \Pi_{t}}(s)\right)-F\left(y_{i, \Pi_{t}}(s)\right)\right] e^{-\int_{t}^{s} \lambda\left[1-F\left(y_{k_{t}, \Pi_{t}}(z)\right)\right] d z}
$$

is the density of the waiting time till the first arrival of an agent with a type in the interval $\left[y_{i, \Pi_{t}}(s), y_{i-1, \Pi_{t}}(s)\right)$ given that no arrival that leads to a sale (e.g., type above $y_{k_{t}}(s)$ ) has occurred.

Recall that a Markovian, non-randomized policy must specify an allocation decision for each possible state, i.e., for each possible subset of object $\Pi_{t} \neq \emptyset$ available at time $t$. Moreover, for each state, the policy consists of $k_{t}=\left|\Pi_{t}\right|$ cut-off curves that describe the partition of the set of agents' types - generally these curves depend on the precise composition of the set $\Pi_{t}$. The number of needed curves if there are $n$ objects is $\sum_{k=1}^{n} k\left(\begin{array}{l}n \\ k\end{array}\right)=n 2^{n-1}$. This yields 4 cut-off curves for two objects, 12 curves for three objects, 32 curves for 4 objects, and so on... In order to save on notation and to keep the somewhat involved proofs more transparent, we assume below that there are only two objects with characteristics $q_{1} \geq q_{2}$. But we will describe the completely analogous solution to the revenue maximization problem for the general case with any number of distinct objects. A main result is that the dynamic revenue maximizing policy for $n$ (possibly distinct) objects is, in fact, completely described by only $n$ cutoff curves. In particular, it shows that this policy is independent of the characteristics of the available objects.

With slight abuse of notation, we write "2" instead of $\Pi_{t}=\left\{q_{1}, q_{2}\right\}$ at the second subscript of the allocation functions $y_{i, \Pi_{t}}(t)$ whenever $k_{t}=2$. This should not lead here to any confusion.

Proposition 2 Assume that the distribution of the agents' valuations satisfies the assumption of increasing virtual types, i.e., the function $x-\frac{1-F(x)}{f(x)}$ is increasing. The dynamic revenue maximizing allocation policy is independent of the qualities of available objects $q_{1}$ and $q_{2}$. In particular, we have:

1. $y_{1, q_{1}}(t)=y_{1, q_{2}}(t)=y_{1,2}(t):=y_{1}(t)$ where $y_{1}(t)$ is a solution of

$$
y_{1}(t)=\frac{1-F\left(y_{1}(t)\right)}{f\left(y_{1}(t)\right)}+\lambda \int_{t}^{T} \frac{\left[1-F\left(y_{1}(s)\right)\right]^{2}}{f\left(y_{1}(s)\right)} d s
$$

2. $y_{2,2}(t):=y_{2}(t)$ is a solution of

$$
y_{2}(t)=\frac{1-F\left(y_{2}(t)\right)}{f\left(y_{2}(t)\right)}+\lambda \int_{t}^{T} \frac{\left[1-F\left(y_{2}(s)\right)\right]^{2}}{f\left(y_{2}(s)\right)} d s-R(1, t)
$$


where

$$
R(1, t)=\lambda \int_{t}^{T} \frac{\left[1-F\left(y_{1}(s)\right)\right]^{2}}{f\left(y_{1}(s)\right)} d s
$$

Proof. See Appendix.

Remark 1 Let us explore in some detail the intuition for the result whereby the optimal cutoffs do not depend on qualities. Assume that there are two available objects $q_{1}>q_{2}$, and that at time $t$ the cutoffs are $y_{1}^{o}>y_{2}^{o}$. Consider the effect of small shift in the highest cut-off from $y_{1}^{o}$ to $y_{1}^{o}+\epsilon$. This shift has any effect only if some agent arrives at $t$. Moreover, the shift has no effect on the expected revenue if the arriving agent has value below $y_{1}^{o}$. If at time $t$ an agent with value $y_{1}^{o}$ arrives, then the shift switches the object he gets from $q_{1}$ to $q_{2}$ which implies that he has to pay $P_{t}^{(2)}\left(\left\{q_{1}, q_{2}\right\}\right)$ instead of $P_{t}^{(1)}\left(\left\{q_{1}, q_{2}\right\}\right)$ - and also switches the object that remains available for the future allocation from $q_{2}$ to $q_{1}$. The infinitesimal effect is

$$
\begin{aligned}
& f\left(y_{1}^{o}\right)\left(P_{t}^{(2)}\left(\left\{q_{1}, q_{2}\right\}\right)+q_{1} R(1, t)-P_{t}^{(1)}\left(\left\{q_{1}, q_{2}\right\}\right)-q_{2} R(1, t)\right) \\
= & \left(q_{1}-q_{2}\right) f\left(y_{1}^{o}\right)\left(R(1, t)-y_{1}^{o}\right)
\end{aligned}
$$

where $R(1, t)$ denotes here the expected revenue at time $t$ if the optimal policy will be followed from time $t$ on. The equality in the above equation follows here from the fact that in any incentive compatible mechanism we must have $P_{t}^{(2)}\left(\left\{q_{1}, q_{2}\right\}\right)-P_{t}^{(1)}\left(\left\{q_{1}, q_{2}\right\}\right)=\left(q_{2}-q_{1}\right) y_{1}^{o}$. Recall also that $P_{t}^{(1)}\left(\left\{q_{1}, q_{2}\right\}\right)=\left(q_{1}-q_{2}\right) y_{1}^{o}+q_{2} y_{2}^{o}$. Therefore, the shift increases the price that will be charged to all agents with type above $y_{1}^{o}+\epsilon$, since supporting a more conservative allocation of the best available object requires charging a higher price to all types that should get this object. Therefore, increasing the cut-off $y_{1}^{o}$ also yields a higher revenue if an agent with value above $y_{1}^{o}+\epsilon$ arrives. This effect is

$$
\begin{aligned}
& \left(1-F\left(y_{1}^{o}+\epsilon\right)\right)\left(\left(q_{1}-q_{2}\right)\left(y_{1}^{o}+\epsilon\right)+q_{2}\left(y_{2}^{o}+R(1, t)\right)\right) \\
& -\left(1-F\left(y_{1}^{o}+\epsilon\right)\right)\left(\left(q_{1}-q_{2}\right) y_{1}^{o}+q_{2}\left(y_{2}^{o}+R(1, t)\right)\right) \\
= & \left(q_{1}-q_{2}\right)\left(1-F\left(y_{1}^{o}+\epsilon\right)\right) \epsilon
\end{aligned}
$$

For an infinitesimal change, this effect becomes $\left(q_{1}-q_{2}\right)\left(1-F\left(y_{1}^{o}\right)\right)$. To sum up, the total effect of the shift on expected revenue is

$$
\left(q_{1}-q_{2}\right)\left[\left(1-F\left(y_{1}^{o}\right)\right)-f\left(y_{1}^{o}\right)\left(y_{1}^{o}-R(1, t)\right)\right] .
$$

The expression is linear in the difference $\left(q_{1}-q_{2}\right)$ and the optimal $y_{1}^{o}$ - where the total effect of the shift should be equal to zero - does not depend on the characteristics of the available objects. This intuition includes the case with only one object, corresponding to $q_{2}=0 .{ }^{16}$

Remark 2 The equations for the revenue maximizing cutoff curves have an intuitive interpretation. Assume first that only one object with $q=1$ is available. The allocation policy is described by the equation

$$
y_{1}(t)-\frac{1-F\left(y_{1}(t)\right)}{f\left(y_{1}(t)\right)}=\lambda \int_{t}^{T} \frac{\left[1-F\left(y_{1}(s)\right)\right]^{2}}{f\left(y_{1}(s)\right)} d s=R(1, t)
$$

\footnotetext{
${ }^{16}$ Another way to describe the intuition is to consider fixed cutoffs, but double, say, the difference between the two qualities. Then, if an agent with type $y_{1}^{o}$ arrives, assigning object 1 rather than 2 yields more revenue, but so does holding object 1 for future allocation. Given the linearity in quality of revenue with one object, these two effects cancel each other.
} 
On the left hand side, we have the virtual valuation of an agent with type $y_{1}(t)$. As Claim 1 showed, the right hand side represents the expected revenue from time $t$ on if the object is not sold at $t$, given that an optimal allocation policy is followed from time $t$ on. Since the seller is able to extract as revenue only the virtual valuation of an arriving buyer, the equation shows that the optimal cut-off curve satisfies an indifference condition between immediate selling and a continuation that uses the optimal policy.

In the general case, if there are $k_{t}=\left|\Pi_{t}\right|$ available objects, then, no matter what their types are, the $i$ 'th cut-off curve, $1 \leq i \leq k_{t}$, in the dynamic revenue-maximizing policy is given by

$$
y_{i}(t)-\frac{1-F\left(y_{i}(t)\right)}{f\left(y_{i}(t)\right)}+\lambda \int_{t}^{T} \frac{\left[1-F\left(y_{i-1}(s)\right)\right]^{2}}{f\left(y_{i-1}(s)\right)} d s=\lambda \int_{t}^{T} \frac{\left[1-F\left(y_{i}(s)\right)\right]^{2}}{f\left(y_{i}(s)\right)} d s
$$

or, equivalently, by

$$
y_{i}(t)-\frac{1-F\left(y_{i}(t)\right)}{f\left(y_{i}(t)\right)}+R\left(\mathbf{1}_{i-1}, t\right)=R\left(\mathbf{1}_{i}, t\right)
$$

where $\mathbf{1}_{i}$ is the set of $1^{\prime} s$ of cardinality $i$ and

$$
R\left(\mathbf{1}_{j}, t\right)=\lambda \int_{t}^{T} \frac{\left[1-F\left(y_{j}(s)\right)\right]^{2}}{f\left(y_{j}(s)\right)} d s
$$

is the expected revenue at time $t$ from the optimal cut-off policy if $j$ identical objects with $q=1$ are still available. Since there will be sales only to the agents with positive virtual valuations, equation (4) implies that $R\left(\mathbf{1}_{i}, t\right)>R\left(\mathbf{1}_{j}, t\right)$ for any $i>j \geq 0$ and $t<T$.

While equation (4) has been obtained for the case of identical objects in the revenuemanagement literature (see for example Gallego and van Ryzin [12], and Bitran and Mondschein [6] for a discrete time model), the explicit expression in (5) is new, a by-product of our analysis that focused on the allocation policy rather than on prices.

For the general case with several distinct objects, note also that, if an object is sold at time $t$, then the lowest among the current optimal cut-off curves becomes irrelevant regardless of the characteristic of the sold object, while all the other $k_{t}-1$ cutoff curves do not change and remain relevant for the future allocation decisions. That is, the optimal cutoff curves depend only on the cardinality of $\Pi_{t}, k_{t}$. For any two sets of available objects $\Pi_{t}^{1}$ and $\Pi_{t}^{2}$ with $k_{t}^{1}=\left|\Pi_{t}^{1}\right|$ and $k_{t}^{2}=\left|\Pi_{t}^{2}\right|$, and for any $1 \leq i \leq \min \left\{k_{t}^{1}, k_{t}^{2}\right\}$ it holds that

$$
y_{i, \Pi_{t}^{1}}(t)=y_{i, \Pi_{t}^{2}}(t) \text {. }
$$

If $\Pi_{t}$ is a set of identical objects, then only the lowest cut-off curve $y_{k_{t}}(t)$ where $k_{t}=\left|\Pi_{t}\right|$ is relevant for the allocation decision.

\section{Applications}

\subsection{Comparison of the Efficient and the Revenue Maximizing Policies}

We use here our previous characterization result in order to compare the revenue-maximizing allocation policy to the efficient one: the curves describing the revenue-maximizing allocation are always above the respective curves describing the efficient allocation if the agents' types follow a distribution with an increasing failure (or hazard) rate - (IFR hereafter). In other words, at any point in time, the revenue maximizing policy is more "conservative".

It should be obvious that our solution method can be also be used to derive the dynamic welfare-maximizing policy along lines analogous to the above. Fortunately, a shortcut is 
available since Albright [1] characterized the welfare-maximizing allocation policy in the complete-information model, and showed that it is independent of the objects' qualities. In his model, the type of the arriving agent becomes public information upon arrival, and prices/implementation issues do not play a role in his analysis. But, since his solution turns out to be a partition-based allocation, Proposition 1 shows that it can be implemented also in our set-up with incomplete information, and thus it can be directly compared to the revenue maximizing policy. Alternatively, the possibility to implement the efficient allocation in our framework can be deduced by general results that focus on dynamic extensions of VCG schemes such as Parkes and Singh [27], Bergemann and Valimäki [5] and Athey and Segal $[3]$.

Proposition 3 Assume that the distribution of values $F$ is IFR, and denote by $y_{i}^{e}(t)\left(y_{i}^{o}(t)\right)$ the efficient (revenue-maximizing) cut-off curve for the object with the $i$ 'th highest characteristic. Then, for any $t \in[0, T], y_{i}^{e}(t)<y_{i}^{o}(t), i=1,2, . . n$.

Proof. See Appendix.

Since the implementation of an allocation policy $\left\{y_{i, \Pi_{t}}(t)\right\}$ dictates the payment scheme

$$
P_{t}^{(j)}\left(\Pi_{t}\right)=\sum_{i=j}^{k_{t}}\left(q_{(i)}-q_{(i+1)}\right) y_{i, \Pi_{t}}(t),
$$

the previous result implies that, for any set of the available objects $\Pi_{t}$, the prices under efficient allocation are also lower then under the revenue maximizing allocation. The next example illustrates the Proposition:

Example 1 Assume that there are two objects, that the arrival process is homogenous with rate $\lambda=1$, and that the distribution of agents' types is exponential, i.e., $F(x)=1-e^{-x}$. The efficient policy is described by the cutoff functions

$$
\begin{aligned}
& y_{1}^{e}(t)=\ln (1+T-t) \\
& y_{2}^{e}(t)=\ln \left(1+\frac{(T-t)^{2}}{2(1+T-t)}\right)
\end{aligned}
$$

while the revenue-maximizing policy is given by

$$
\begin{aligned}
& y_{1}^{o}(t)=\ln (e+T-t) \\
& y_{2}^{o}(t)=\ln \left(\frac{1}{2}(e+T-t)+\frac{1}{2} \frac{e^{2}}{(e+T-t)}\right)
\end{aligned}
$$

The following figure plots the solutions for $T=5$. The solid lines represent the revenue maximizing cut-off curves and the dashed lines represent the efficient cut-off curves. 


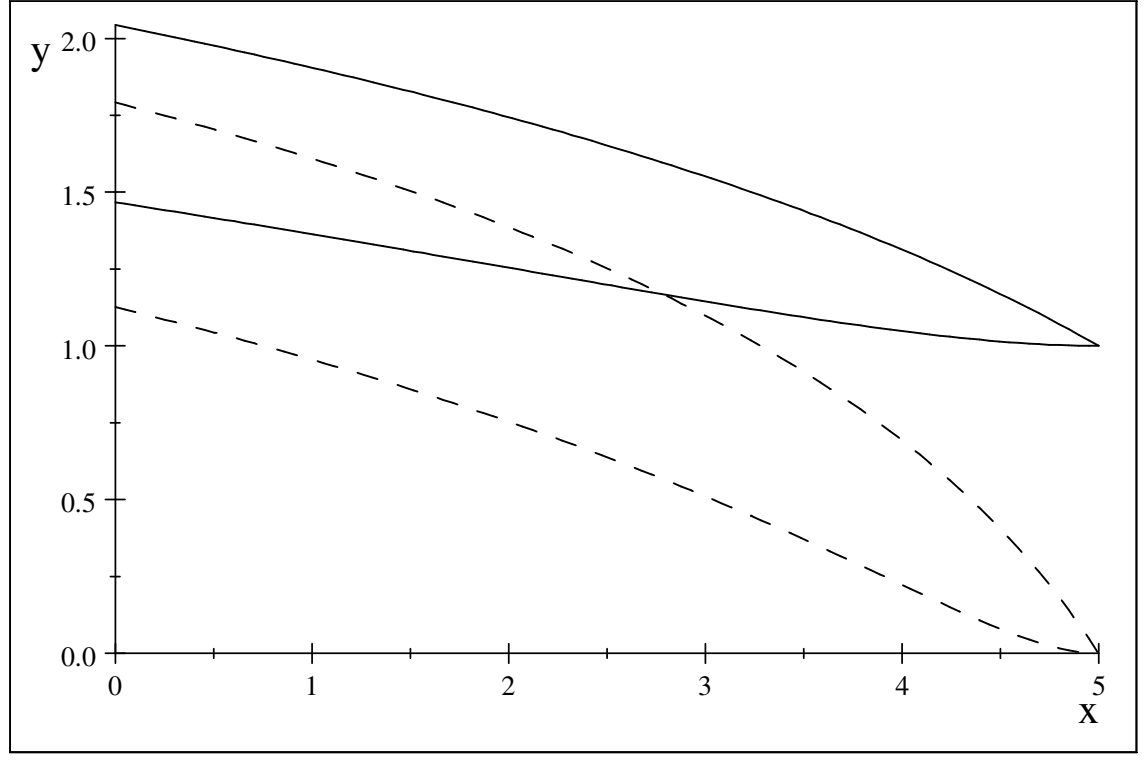

Our last example in this Subsection shows that the efficient and revenue-maximizing cutoff curves may coincide if the IFR assumption is not satisfied. In particular, an increasing virtual valuation is not sufficient for the above result.

Example 2 (McAfee and te Velde [20]) Assume that there is one object, that the arrival process is Poisson with rate $\lambda=1$, and that values are distributed according to a Pareto distribution, that is $F(x)=1-x^{-\epsilon}$ for $x \geq 1, \epsilon>1$.

The failure rate is given by $\frac{f(x)}{1-F(x)}=\frac{\epsilon}{x}$ which is of course decreasing. McAfee and te Velde showed for this case that the efficient and revenue-maximizing cutoff curves coincide:

$$
y_{1}^{e}(t)=y_{1}^{o}(t)=\left[\frac{\epsilon}{\epsilon-1}(T-t)\right]^{\frac{1}{\epsilon}} .
$$

Note also that the virtual valuation is given by $x-\frac{1-F(x)}{f(x)}=x\left(1-\frac{1}{\epsilon}\right)$, which is increasing in $x$ since $\epsilon>1$. This shows that an increasing virtual valuation is not sufficient for the result of Proposition 3.

\subsection{Dynamic Pricing and Optimal Inventory Choice}

The entire above analysis was based on the assumption that the inventory's size and composition was exogenously given. In this Subsection we show how our previous insights can be immediately used as a building block for a more general analysis where the seller can choose both the number of objects and the spectrum of offered qualities before sales start. This timing assumption is appropriate in cases where the necessary production lead-times are significant in comparison to the retail period. Our present treatment extends the seminal Mussa-Rosen [24] analysis to the framework with random arrivals. Gallego\&van Ryzin characterized the optimal inventory size in a model with deterministic demand, and showed that the solution coincides with the optimal size of the inventory in the original problem if the horizon goes to infinity. In contrast, we are able to characterize the optimal inventory size (and its composition) in the original problem for any finite horizon. 
Since our analysis identified an invariant of the optimal selling scheme - the allocation policy - we can easily solve the larger problem backwards and characterize the optimal quality choice. Formally, the decision of the monopolist is to choose the optimal package of qualities $\Pi_{0}$ :

$$
\max _{\Pi_{0}} R\left(\Pi_{0}, 0\right)-C\left(\Pi_{0}\right)
$$

where $C(\Pi)$ denotes cost of producing the package of qualities $\Pi$. It is plausible to assume that the cost function is convex and symmetric (symmetry means here that the cost of producing any permutation of a given package of qualities is the same as the cost of producing the original package). Note that any convex and symmetric function is Schur-convex. ${ }^{17}$ We assume below a very simple, separable form of Schur-convexity for the cost function, but the reader will have no difficulty adding the technical details needed for the more general result that does not assume separability.

Proposition 4 Let $y=\left\{y_{i}(t)\right\}_{i=1}^{n}$ denote the allocation underlying the revenue maximizing policy with $n$ objects, and assume that, for all $n, C\left(q_{1}, q_{2}, . . q_{n}\right)=\sum_{i=1}^{n} \phi\left(q_{i}\right)$ where the function $\phi: R \rightarrow R$ is strictly increasing, convex and satisfies $\phi(0)=0$. Then :

1. The optimal number of objects $n^{*}$ is characterized by ${ }^{18}$

$$
\phi^{\prime}(0) \in\left(y_{n^{*}+1}(0)-\frac{1-F\left(y_{n^{*}+1}(0)\right)}{f\left(y_{n^{*}+1}(0)\right)}, y_{n^{*}}(0)-\frac{1-F\left(y_{n^{*}}(0)\right)}{f\left(y_{n^{*}}(0)\right)}\right]
$$

2. The optimal qualities $q_{i}^{*}$ are given by:

$$
\phi^{\prime}\left(q_{i}^{*}\right)=y_{i}(0)-\frac{1-F\left(y_{i}(0)\right)}{f\left(y_{i}(0)\right)}, i=1, \ldots, n^{*}
$$

Proof. See Appendix.

Together with the result of the previous Section, the above proposition and its analog for welfare maximization can be used to compare the quality/quantity optimal choices under the two regimes. For example, it is intuitive that a revenue maximizing monopolist will produce lower qualities than a welfare maximizing one, as in the Mussa-Rosen framework. Note though that some insights will differ from Mussa and Rosen's due to a difference in timing: while we assume that qualities are produced in advance - before buyers' characteristics get revealed - Mussa and Rosen implicitly assume that quality can be produced contigent on these. For instance, in our setup there is a distortion even in the quality provided to the highest type.

\subsection{The Pattern of Prices in Clearance Sales}

Our characterization generates a wealth of empirically testable implications about the pattern of observed prices associated with the revenue maximizing policy. These implications can be compared to available data for relevant situations. For example, an important finding in the well-known empirical study of clearance sales for apparel conducted by Pashigian and Bowen [29] is that :

\footnotetext{
${ }^{17}$ Schur-convexity guarantees here that a higher marginal revenue is associated with a higher chosen quality for the respective object.

${ }^{18}$ It should be obvious how to adjust the formula in order to deal with the extreme cases where $n^{*}=0, \infty$
} 
"More expensive apparel items within each product line are frequently sold at a higher average percentage markdown".

Pashigian and Bowen attempt to explain this phenomenon by using Lazear's [18] theory of retail pricing and clearance sales. Lazear's theory only deals with the sale of a homogenous product and it cannot incorporate the parallel sale of several substitutes, as is common practice in most stores. Thus, the offered theoretical explanation for the empirical finding is not entirely convincing ${ }^{19}$. The next result shows how the empirical observation agrees with a fairly general prediction of our model that holds for any two different qualities, for any positive levels of inventories before and after the clearance sale, and for any distribution of values.

Proposition 5 Assume that the seller uses the revenue maximizing policy in a situation where at time $t=0$ there are $n_{1}>0$ items of quality $q$ and $n_{2}>0$ items of quality $s, s<q$. Assume also that at time $t=T$ there are $0<l_{1} \leq n_{1}$ items of quality $q$, and $0<l_{2} \leq n_{2}$ items of quality s left unsold. Then the percentage markdown - defined as the difference between the prices of the same product at $t=0$ and $t=T$ divided by the price at $t=0$ - is always higher for the higher quality.

Proof. See Appendix.

We conclude this subsection with another related observation that can also be taken to the data:

Proposition 6 Consider the revenue maximizing policy. Then, at any point in time $t$ where two different qualities are available, the price of the higher quality decreases quicker than the price of the lower quality.

Proof. See Appendix.

\section{Conclusion}

We have studied the dynamic, revenue maximizing allocation of finite inventories consisting of substitute qualities to a stream of privately informed agents that arrive randomly before a deadline. The paper's main contributions were:

1. The integrated model considerably broadens the scope of the optimization exercise, and allows us to add quantity/quality decisions to the traditional pricing considerations for identical objects.

2. We have introduced a new methodology - based on a combination of the payoff equivalence principle and a variational argument - to the analysis of inventory pricing under random, sequential demand.

3. The analysis yields clear, testable implications about the pattern of optimal evolution of prices over time. These predictions can be taken to the data.

\footnotetext{
${ }^{19}$ For some special forms of the distribution of values, Lazear's theory predicts that increasing the dispersion in values for the product will lead to an increased markdown. Pashigian and Bowen identify more expensive items (which contain more elements of fashion and style) with an increased dispersion.
} 
An important assumption has been that agents' purchase decisions are not strategic in their time dimension. Of course, if all agents can delay their purchase till the deadline without cost, the optimal mechanism will be an auction that gathers all potential buyers - this is the opposite extreme assumption to the one made in this paper where buyers are infinitely impatient. It is of major theoretical and applied interest to study revenue maximizing schemes that take into account the agents' incentives to possibly delay their transactions in less extreme cases. Since, as sales occur, prices may go up and inventories get depleted, the decision whether to delay purchase to a later time is complex. The same applies to the seller's reaction to such buyer behavior which may need to include mechanisms that are more complex than menus of posted prices or standard auctions.

Other major - but also difficult - generalizations would be the introduction of heterogeneous objects that are not necessarily similarly ranked by all buyers, or the introduction of multi-unit demand. The latter would allow the study of dynamic bundling policies. Such models need a multi-dimensional specification of private information.

Finally, our analysis has considered a monopolist seller, but it would be very interesting to characterize dynamic pricing equilibria among oligopolists that face randomly arriving buyers who can decide where to buy.

\section{References}

[1] Albright S.C. (1974): "Optimal Sequential Assignments with Random Arrival Times", Management Science $\mathbf{2 1}$ (1), 60-67.

[2] Arnold, M.A. and Lippman, S.A. (2001): "The Analytics of Search with Posted Prices", Economic Theory 17, 444-466.

[3] Athey, S. and Segal, Y (2007): "An Efficient Dynamic Mechanism", discussion paper, Stanford University.

[4] Aviv, Y. and Pazgal, A (2007): "Optimal Pricing of Seasonal Products in the Presence of Forward-Looking Consumers", Manufacture $\&$ Service Operations Management, forthcoming

[5] Bergemann, D. and Välimäki, J. (2006): "Efficient Dynamic Auctions", Cowles Foundation Discussion Paper No. 1584

[6] Bitran, G.R. and Mondschein, S.V. (1997): "Periodic Pricing of Seasonal Products in Retailing", Management Science 43 (1), 64-79.

[7] Bitran, G.R. and Caldentey, R. (2003): "An Overview of Pricing Models for Revenue Management", Manufacture ES Services Operations Management 5(3), 203-229.

[8] Bulow, J. and Roberts, J. (1989): "The Simple Economics of Optimal Auction", Journal of Political Economy 97 (5), 1060-1090.

[9] Derman, C., Lieberman, G.J., and Ross, S.M. (1972): "A Sequential Stochastic Assignment Process", Management Science 18 (7), 349-355.

[10] Das Varma, G., and Vettas, N. (2001): "Optimal Dynamic Pricing with Inventories", Economic Letters 72 (3), 335-340. 
[11] Elmaghraby, W. and Keskinocak, P. (2003): "Dynamic Pricing in the Presence of Inventory Considerations: Research Overview, Current Practices, and Future Directions", Management Science 49 (10), 1287-1309.

[12] Gallego, G. and van Ryzin, G. (1994): "Optimal Dynamic Pricing of Inventories with Stochastic Demand over Finite Horizons", Management Science 40 (8), 999-1020.

[13] Gallego, G. and van Ryzin, G. (1997): "A Multiproduct Dynamic Pricing Problem and its Applications to Network Yield Management", Operations Research 45 (1), 24-41.

[14] Gallien, J. (2006): "Dynamic Mechanism Design for Online Commerce", Operations Research 54 (2), 291-310.

[15] Gihman, I.I. and Skorohod, A.V. (1979): Controlled Stochastic Processes, Springer: New York

[16] Harris, M. and Raviv, A. (1981): "A Theory of Monopoly Pricing Schemes with Deman Uncertainty", American Economic Review $\mathbf{7 1}$ (3), 347-365.

[17] Kincaid, W.M., and Darling, D. (1963): "An Inventory Pricing Problem", Journal of Mathematical Analysis and Applications 7, 183-208.

[18] Lazear, E.P. (1986): "Retail Pricing and Clearance Sale", The American Economic Review 76 (1), 14-32.

[19] Lippman, S.A. and McCall, J. (1981): "The Economics of Uncertainty: Selected Topics and Probabilistic Methods", in Handbook of Mathematical Economics, Ed. K.J. Arrow and M.D. Intriligator, Volume I, Elsevier, 211-284.

[20] McAfee, P. and te Velde, V. (2007a): "Dynamic Pricing with Constant Demand Elasticity", Production and Operations Management, Special Issue on Revenue Management and Dynamic Pricing, forthcoming.

[21] McAfee, P. and te Velde, V. (2007b): "Dynamic Pricing in the Airline Industry", in Handbook on Economics and Information Systems, Ed: T.J. Hendershott, Elsevier Handbooks in Information Systems, Volume I.

[22] McAfee, P. and Wiseman, T. (2008): "Capacity Choice Counters the Coase Conjecture", Review of Economic Studies $\mathbf{7 5}$ (1), 317-332.

[23] Mortensen, D.T. (1986): "Job Search and Labor Market" in Handbook of Labor Economics, Ed: O. Ashenfelter and R. Layard, Volume II, Elsevier, 849-919

[24] Mussa, M. and Rosen, S. (1978): "Monopoly and Product Quality", Journal of Economic Theory 18, 301-317

[25] Myerson, R. (1981): "Optimal Auction Design," Mathematics of Operation Research 6, 58-73.

[26] Nocke, V. and Peitz, M (2007): "A Theory of Clearance Sales," Economic Journal 117, 964-990

[27] Parkes, D.C., and Singh, S. (2003): "An MDP-Based Approach to Online Mechanism Design", Proceedings of 17th Annual Conference on Neural Information Processing Systems (NIPS 03) 
[28] Pashigian, B.P. (1988): "Demand Uncertainty and Sales: A Study of Fashion and Markdown Prices", The American Economic Review 78 (5) , 936-953.

[29] Pashigian, B.P. and Bowen, B. (1991), "Why Are Products Sold on Sale?: Explanations of Pricing Regularities", The Quarterly Journal of Economics 106 (4), 1015-1038

[30] Pashigian, B.P., Bowen, B and Gould, E. (1995), "Fashion, Styling, and the WithinSeason Decline in Automobile Prices", Journal of Law and Economics 38 (2), 281-309.

[31] Puterman, M.L. (2005): Markov Decision Processes, Wiley: Hoboken, NJ

[32] Riley, J. and Zeckhauser, R. (1983): "Optimal Selling Strategies: When to Haggle, When to Hold Firm", The Quarterly Journal of Economics, 98(2), 267-289.

[33] Ross, S.M. (1983): Stochastic Processes, Wiley: New York.

[34] Su, Xuanming (2006): "Inter-temporal Pricing with Strategic Customer Behavior", discussion paper, Haas School of Business, University of California, Berkeley

[35] Talluri, K.T., and Van Ryzin, G. (2004): The Theory and Practice of Revenue Management, Springer: New York.

[36] Wang, R. (1993): "Auctions versus Posted-Price Selling", The American Economic Review 83 (4), 838-851.

[37] Wilson, C. A. (1988): "On the Optimal Pricing Policy of a Monopolist", Journal of Political Economy 96 (1), 164-176.

\section{Appendix}

Proof of Proposition 2: The proof proceeds by a sequence of three arguments: first, we derive the expected revenue for any Markovian, non-randomized allocation policy (Proposition 7 ); second, we derive the revenue-maximizing cutoff curves when only one object remains (Claim 1); finally, we derive the revenue maximizing allocation policy if two objects are left (Claim 2).

\section{Proposition 7 Assume that}

1. If $k_{t}=2$, the designer uses the dynamic allocation cutoff-curves $y_{2,2}(t) \leq y_{1,2}(t)$, i.e., the agent that arrives at time $t$ gets: the object with quality $q_{1}$ if his type is $x_{i} \geq y_{1,2}(t)$; the object with quality $q_{2}$, if his type is $x_{i} \in\left[y_{2,2}(t), y_{1,2}(t)\right)$; no object if $x_{i}<y_{2,2}(t)$.

2. If $k_{t}=1$, the designer uses the dynamic cutoff-curves $y_{1, q_{j}}(t)$, i.e., the agent that arrives at time $t$ gets the remaining object with characteristic $p_{j}$ if $x_{i} \geq y_{1, q_{j}}(t)$, and no object otherwise.

Then, the expected revenue from this policy is given by

$$
\begin{aligned}
& \int_{0}^{T}\left(q_{2} y_{2,2}(t)+R\left(q_{1}, t\right)\right) \lambda\left(1-F\left(y_{2,2}(t)\right)\right) e^{-\int_{0}^{t} \lambda\left(1-F\left(y_{2,2}(s)\right)\right) d s} d t+ \\
& \int_{0}^{T}\left(\left(q_{1}-q_{2}\right) y_{1,2}(t)+R\left(q_{2}, t\right)-R\left(q_{1}, t\right)\right) . \\
& \lambda\left(1-F\left(y_{1,2}(t)\right)\right) e^{-\int_{0}^{t} \lambda\left(1-F\left(y_{2,2}(s)\right)\right) d s} d t
\end{aligned}
$$


where

$$
R\left(q_{j}, t\right)=q_{j} \int_{t}^{T} y_{1, q_{j}}(s) \lambda\left(1-F\left(y_{1, q_{j}}(s)\right) e^{-\int_{t}^{s} \lambda\left[1-F\left(y_{1, q_{j}}(z)\right)\right] d z} d s\right.
$$

is the expected revenue at time $t$ if only one object with quality $q_{j}$ remains, given that the dynamic allocation function $y_{1, q_{j}}$ is used from $t$ on.

Proof of Proposition 7. If only one object with characteristic $q_{i}$ is available at time $t$, then the expected revenue is given by

$$
q_{i} \int_{t}^{T} y_{1, p_{i}}(s) h_{1, q_{i}}(s) d s
$$

where $h_{1, q_{i}}(s)$ represents the density of the waiting time till the first arrival of an agent with a value that is at least $y_{1, q_{i}}(s)$. Note that this density is equal to the density of the first arrival in a non-homogenous Poisson process with rate $\lambda(s)\left(1-F\left(y_{1, q_{i}}(s)\right)\right.$. The density of the time of the $n$-th arrival in a non-homogenous Poisson process with rate $\delta(s)$ is given by (see Ross [33])

$$
g_{n}(s)=\delta(s) e^{-m(s)} \frac{m(s)^{n-1}}{(n-1) !}, \text { where } m(s)=\int_{t}^{s} \delta(z) d z
$$

Thus, in our case, we obtain

$$
h_{1, q_{i}}(s)=\lambda(s)\left(1-F\left(y_{1, q_{i}}(s)\right) e^{-\int_{t}^{s} \lambda(z)[1-F(y(z))] d z} \text { for } t \leq s \leq T\right.
$$

and (8) follows.

If two objects are still available, the expected revenue is given by

$$
\int_{0}^{T}\left[P_{t}^{(2)}\left(\left\{q_{1}, q_{2}\right\}\right)+R\left(q_{1}, t\right)\right] h_{2,2}(t) d t+\int_{0}^{T}\left[P_{t}^{(1)}\left(\left\{q_{1}, q_{2}\right\}\right)+R\left(q_{2}, t\right)\right] h_{1,2}(t) d t
$$

Here $h_{1,2}(t)$ represents the density of the waiting time till the first arrival of an agent with a value that is at least $y_{1,2}(t)$ if no arrival of an agent with value in the interval $\left[y_{2,2}(t), y_{1,2}(t)\right)$ has occurred. Similarly, $h_{2,2}(t)$ represents the density of the waiting time till the first arrival of an agent with a value in the interval $\left[y_{2,2}(t), y_{1,2}(t)\right)$ if no arrival of an agent with value in the interval $\left[y_{1,2}(t), \infty\right)$ has occurred. Since the arrival processes of agents with types in the intervals $\left[y_{2,2}(t), y_{1,2}(t)\right)$ and $\left[y_{1,2}(t), \infty\right)$, respectively, are independent non-homogenous Poisson processes (see Proposition 2.3.2 in Ross [33] ), using (9) we obtain

$$
\begin{aligned}
h_{1,2}(t) & =\lambda\left(1-F\left(y_{1,2}(t)\right)\right) e^{-\int_{0}^{t} \lambda\left[1-F\left(y_{1,2}(s)\right)\right] d s} e^{-\int_{0}^{t} \lambda\left[F\left(y_{1,2}(s)\right)-F\left(y_{2,2}(s)\right)\right] d s} \\
& =\lambda\left(1-F\left(y_{1,2}(t)\right)\right) e^{-\int_{0}^{t} \lambda\left[1-F\left(y_{2,2}(s)\right)\right] d s}
\end{aligned}
$$

and

$$
\begin{aligned}
h_{2,2}(t) & =\lambda\left(F\left(y_{1,2}(t)\right)-F\left(y_{2,2}(t)\right)\right) e^{-\int_{0}^{t} \lambda\left[F\left(y_{1,2}(s)\right)-F\left(y_{2,2}(s)\right)+1-F\left(y_{1,2}(s)\right)\right] d s} \\
& =\lambda\left(F\left(y_{1,2}(t)\right)-F\left(y_{2,2}(t)\right)\right) e^{-\int_{0}^{t} \lambda\left[1-F\left(y_{2,2}(s)\right)\right] d s}
\end{aligned}
$$

Finally, recall that incentive compatibility implies that

$$
P_{t}^{(2)}\left(\left\{q_{1}, q_{2}\right\}\right)=q_{2} y_{2,2}(t) \text { and } P_{t}^{(1)}\left(\left\{q_{1}, q_{2}\right\}\right)=q_{2} y_{2,2}(t)+\left(q_{1}-q_{2}\right) y_{1,2}(t),
$$

Plugging the expressions for $P_{t}^{(2)}\left(\left\{q_{1}, q_{2}\right\}\right), P_{t}^{(1)}\left(\left\{q_{1}, q_{2}\right\}\right), h_{1,2}(t)$ and $h_{2,2}(t)$ into the expression for expected revenue (10) yields the required formula. 
Claim 1 If only one object remains, the dynamic revenue maximizing allocation curve $y_{1}(t)$ solves

$$
y_{1}(t)=\frac{1-F\left(y_{1}(t)\right)}{f\left(y_{1}(t)\right)}+\lambda \int_{t}^{T} \frac{\left[1-F\left(y_{1}(s)\right)\right]^{2}}{f\left(y_{1}(s)\right)} d s .
$$

The expected revenue at time $t$ where $\Pi_{t}=q_{j}$ is given by $R\left(q_{j}, t\right)=q_{j} R(1, t)$ where

$$
R(1, t)=\lambda \int_{t}^{T} \frac{\left[1-F\left(y_{1}(s)\right)\right]^{2}}{f\left(y_{1}(s)\right)} d s
$$

Proof of Claim 1. If only the object with characteristic $q_{j}$ is available, it follows from Proposition 7 that the expected revenue at time $t$ is given by

$$
R\left(q_{j}, t\right)=q_{j} \int_{t}^{T} y_{1, q_{j}}(s) \lambda\left(1-F\left(y_{1, q_{j}}(s)\right) e^{-\int_{t}^{s} \lambda\left[1-F\left(y_{1, q_{j}}(z)\right)\right] d z} d s .\right.
$$

Let $H(s)=\int_{t}^{s} \lambda\left[1-F\left(y_{1, q_{j}}(z)\right)\right] d z$. Then, we obtain

$$
R\left(q_{j}, t\right)=q_{j} \int_{t}^{T} F^{-1}\left[1-\frac{H^{\prime}(s)}{\lambda}\right] H^{\prime}(s) e^{-H(s)} d s .
$$

This expression for revenue is appropriate for using a variational argument with respect to the function $H$. The corresponding necessary condition for the variational problem (i.e., the Euler-Lagrange equation) is

$$
-\left(H^{\prime}(s)\right)^{2}+2 H^{\prime \prime}(s)+\frac{H^{\prime}(s) H^{\prime \prime}(s) f^{\prime}\left(F^{-1}\left(1-\frac{H^{\prime}(s)}{\lambda}\right)\right)}{\left(f\left(1-\frac{H^{\prime}(s)}{\lambda}\right)\right)^{2}}=0
$$

Plugging back the expression for $H(s)$ gives

$$
-\lambda\left[1-F\left(y_{1, q_{j}}(s)\right)\right]^{2}-2 f\left(y_{1, q_{j}}(s)\right) y_{1, q_{j}}^{\prime}(s)-\frac{\left[1-F\left(y_{1, q_{j}}(s)\right)\right] f^{\prime}\left(y_{1, q_{j}}(s)\right) y_{1, q_{j}}^{\prime}(s)}{f\left(y_{1, q_{j}}(s)\right)}=0
$$

This implies that for any $s \in[0, T]$, the solution $y_{1, q_{j}}(s)$ should satisfy

$$
-y_{1, q_{j}}^{\prime}(s)-y_{1, q_{j}}^{\prime}(s)\left(1+\frac{\left(1-F\left(y_{1, q_{j}}(s)\right)\right) f^{\prime}\left(y_{1, q_{j}}(s)\right)}{\left(f\left(y_{1, q_{j}}(s)\right)\right)^{2}}\right)=\lambda \frac{\left(1-F\left(y_{1, q_{j}}(s)\right)\right)^{2}}{f\left(y_{1, q_{j}}(s)\right)}
$$

Since for any $t$, and for any differentiable $y(t)$ it holds that

$$
-y^{\prime}(t)\left(1+\frac{(1-F(y(t))) f^{\prime}(y(t))}{(f(y(t)))^{2}}\right)=\frac{d}{d t}\left(\frac{1-F(y(t))}{f(y(t))}\right),
$$

we can rewrite the necessary condition as

$$
y_{1, q_{j}}^{\prime}(s)+\lambda \frac{\left(1-F\left(y_{1, q_{j}}(s)\right)\right)^{2}}{f\left(y_{1, q_{j}}(s)\right)}=\frac{d}{d s}\left(\frac{1-F\left(y_{1, q_{j}}(s)\right)}{f\left(y_{1, q_{j}}(s)\right)}\right)
$$

Taking now the integral between $t$ and $T$ yields

$$
\begin{aligned}
& \int_{t}^{T} y_{1, q_{j}}^{\prime}(s) d s+\lambda \int_{t}^{T} \frac{\left(1-F\left(y_{1, q_{j}}(s)\right)\right)^{2}}{f\left(y_{1, q_{j}}(s)\right)} d s \\
= & \int_{t}^{T} \frac{d}{d s}\left(\frac{1-F\left(y_{1, q_{j}}(s)\right)}{f\left(y_{1, q_{j}}(s)\right)}\right) d s
\end{aligned}
$$


This is equivalent to:

$$
\begin{aligned}
& y_{1, q_{j}}(T)-y_{1, q_{j}}(t)+\lambda \int_{t}^{T} \frac{\left(1-F\left(y_{1, q_{j}}(s)\right)\right)^{2}}{f\left(y_{1, q_{j}}(s)\right)} d s \\
= & \frac{1-F\left(y_{1, q_{j}}(T)\right)}{f\left(y_{1, q_{j}}(T)\right)}-\frac{1-F\left(y_{1, q_{j}}(t)\right)}{f\left(y_{1, q_{j}}(t)\right)}
\end{aligned}
$$

Together with the boundary condition

$$
y_{1, q_{j}}(T)-\frac{1-F\left(y_{1, q_{j}}(T)\right)}{f\left(y_{1, q_{j}}(T)\right)}=0
$$

we get (11). The assumptions of increasing virtual type and finite density ensure that a solution to (11) exists for any $t$.

To complete the proof and obtain the expression for revenue (12), note that the expected revenue is given by $R\left(q_{j}, t\right)=q_{j} R(1, t)$ where

$$
R(1, t)=\int_{t}^{T} y_{1}(s) \lambda\left(1-F\left(y_{1}(s)\right) e^{-\int_{t}^{s} \lambda\left[1-F\left(y_{1}(z)\right)\right] d z} d s\right.
$$

Differentiating the above with respect to $t$ gives

$$
R^{\prime}(1, t)=\lambda\left(1-F\left(y_{1}(t)\right)\left(R(1, t)-y_{1}(t)\right)\right.
$$

It is then straightforward to verify that the function $\int_{t}^{T} \frac{\left[1-F\left(y_{1}(s)\right)\right]^{2}}{f\left(y_{1}(s)\right)} d s$ satisfies the above differential equation with the boundary condition $R(1, T)=0$.

We proceed now to characterize the revenue-maximizing allocation policy if there are two objects left.

Claim 2 If two objects remain, the dynamic revenue maximizing policy is characterized by two cutoff curves, $y_{1}(t)$ and $y_{2}(t)$, where $y_{1}(t)$ satisfies equation (11) and where $y_{2}(t)$ satisfies:

$$
y_{2}(t)=\frac{1-F\left(y_{2}(t)\right)}{f\left(y_{2}(t)\right)}+\lambda \int_{t}^{T} \frac{\left[1-F\left(y_{2}(s)\right)\right]^{2}}{f\left(y_{2}(s)\right)} d s-R(1, t)
$$

Moreover, the expected revenue at time $t$ for the case $\Pi_{t}=\{1,1\}$ is given by

$$
R(\{1,1\}, t)=\lambda \int_{t}^{T} \frac{\left[1-F\left(y_{2}(s)\right)\right]^{2}}{f\left(y_{2}(s)\right)} d s .
$$

Proof of Claim 2. I. We consider first the case where $q_{1}>q_{2}$. That is, the seller needs to specify two different prices, and hence two different cutoff curves, $y_{1,2}(t)$ and $y_{2,2}(t)$. We can re-write the expected revenue given by Proposition 7 as

$$
\begin{aligned}
& \int_{0}^{T}\left(q_{1} F^{-1}\left(1-\frac{H^{\prime}(t)}{\lambda}\right)+q_{2} R(1, t)\right) H^{\prime}(t) e^{-H(t)} d t \\
& +\left(q_{2}-q_{1}\right) \int_{0}^{T}\left[F^{-1}\left(1-\frac{G^{\prime}(t)}{\lambda}\right)-R(1, t)\right] G^{\prime}(t) e^{-H(t)} d t
\end{aligned}
$$


where

$$
\begin{aligned}
& \int_{0}^{t} \lambda\left[1-F\left(y_{2,2}(s)\right)\right] d s:=H(t) \\
& \int_{0}^{t} \lambda\left[1-F\left(y_{1,2}(s)\right)\right] d s:=G(t) .
\end{aligned}
$$

The necessary conditions for the variational problem (i.e., the Euler-Lagrange equation) with respect to the functions $H(t)$ and $G(t)$, respectively, are:

$$
\begin{gathered}
-\left(q_{2}-q_{1}\right) G^{\prime}(t)\left[F^{-1}\left(1-\frac{G^{\prime}(t)}{\lambda}\right)-R(1, t)\right]-q_{1} \frac{\frac{1}{\lambda}\left(H^{\prime}(t)\right)^{2}}{f\left(F^{-1}\left(1-\frac{H^{\prime}(t)}{\lambda}\right)\right)} \\
-2 q_{1} \frac{\frac{1}{\lambda} H^{\prime \prime}(t)}{f\left(F^{-1}\left(1-\frac{H^{\prime}(t)}{\lambda}\right)\right)}+q_{2} R^{\prime}(1, t)-q_{1} \frac{\frac{1}{\lambda^{2}} H^{\prime}(t) H^{\prime \prime}(t) f^{\prime}\left(F^{-1}\left(1-\frac{H^{\prime}(t)}{\lambda}\right)\right)}{\left[f\left(F^{-1}\left(1-\frac{H^{\prime}(t)}{\lambda}\right)\right)\right]^{3}}=0
\end{gathered}
$$

and

$$
\begin{aligned}
& -\frac{2 \frac{1}{\lambda} G^{\prime \prime}(t)}{f\left(F^{-1}\left(1-\frac{G^{\prime}(t)}{\lambda}\right)\right)}-R^{\prime}(1, t)-\frac{\frac{1}{\lambda^{2}} G^{\prime}(t) G^{\prime \prime}(t) f^{\prime}\left(F^{-1}\left(1-\frac{G^{\prime}(t)}{\lambda}\right)\right)}{\left[f\left(F^{-1}\left(1-\frac{G^{\prime}(t)}{\lambda}\right)\right)\right]^{3}} \\
& -H^{\prime}(t)\left[-\frac{\frac{1}{\lambda} G^{\prime}(t)}{f\left(F^{-1}\left(1-\frac{G^{\prime}(t)}{\lambda}\right)\right)}+F^{-1}\left(1-\frac{G^{\prime}(t)}{\lambda}\right)-R(1, t)\right]=0 .
\end{aligned}
$$

Plugging the expressions for $H(t)$ and $G(t)$ allows us to write the necessary conditions in the following way:

$$
\begin{gathered}
-\left(q_{2}-q_{1}\right) \lambda\left[1-F\left(y_{1,2}(t)\right)\right]\left(y_{1,2}(t)-R(1, t)\right)-q_{1} \frac{\lambda\left[1-F\left(y_{2,2}(t)\right)\right]^{2}}{f\left(y_{2,2}(t)\right)} \\
-2 q_{1} y_{2,2}^{\prime}(t)-q_{2} R^{\prime}(1, t)-q_{1} \frac{y_{2,2}^{\prime}(t)\left[1-F\left(y_{2,2}(t)\right)\right] f^{\prime}\left(y_{2,2}(t)\right)}{\left[f\left(y_{2,2}(t)\right)\right]^{2}}=0
\end{gathered}
$$

and

$$
\begin{gathered}
{\left[1-F\left(y_{2,2}(t)\right)\right]\left[\frac{1-F\left(y_{1,2}(t)\right)}{f\left(y_{1,2}(t)\right)}-y_{1,2}(t)+R(1, t)\right]-2 y_{1,2}^{\prime}(t)} \\
-R^{\prime}(1, t)+\frac{y_{1,2}^{\prime}(t)\left[1-F\left(y_{1,2}(t)\right)\right] f^{\prime}\left(y_{1,2}(t)\right)}{\left[f\left(y_{1,2}(t)\right)\right]^{2}}=0
\end{gathered}
$$

Next, we show that a solution to the system of differential equations 16 and 17 is given by $y_{1,2}(t)=y_{1}(t)$ and $y_{2,2}(t)=y_{2}(t)$ where $y_{1}(t)$ and $y_{2}(t)$ solve the system of equations:

$$
\begin{aligned}
& y_{1}(t)=\frac{1-F\left(y_{1}(t)\right)}{f\left(y_{1}(t)\right)}+\lambda \int_{t}^{T} \frac{\left[1-F\left(y_{1}(s)\right)\right]^{2}}{f\left(y_{1}(s)\right)} d s \\
& y_{2}(t)=\frac{1-F\left(y_{2}(t)\right)}{f\left(y_{2}(t)\right)}+\lambda \int_{t}^{T} \frac{\left[1-F\left(y_{2}(s)\right)\right]^{2}}{f\left(y_{2}(s)\right)} d s-R(t),
\end{aligned}
$$

Again, the assumptions of increasing virtual types and finite density guarantee the existence of solutions for (18) and (19). Note also that, since $R(t) \geq 0$, we must have $y_{2}(t) \leq y_{1}(t)$, $\forall t$. 
Differentiation of (18) with respect to $t$ gives

$$
2 y_{1}^{\prime}(t)=-y_{1}^{\prime}(t) \frac{\left[1-F\left(y_{1}(t)\right)\right] f^{\prime}\left(y_{1}(t)\right)}{\left[f\left(y_{1}(t)\right)\right]^{2}}-\lambda \frac{\left[1-F\left(y_{1}(t)\right)\right]^{2}}{f\left(y_{1}(t)\right)} .
$$

Plugging the above expression into (17), and using the fact that

$$
R^{\prime}(1, t)=-y_{1}(t) \lambda\left(1-F\left(y_{1}(t)\right)\right)+\lambda\left(1-F\left(y_{1}(t)\right)\right) R(1, t)
$$

yields

$$
\left[\lambda \int_{t}^{T} \frac{\left[1-F\left(y_{1}(s)\right)\right]^{2}}{f\left(y_{1}(s)\right)} d s-R(1, t)\right]\left[\lambda\left(1-F\left(y_{1}(t)\right)\right)-\left(1-F\left(y_{2,2}(t)\right)\right)\right]=0
$$

where last equality follows from Claim 1 . Thus, we have showed that $y_{1,2}(t)=y_{1}(t)$ solves (17) for any $y_{2,2}(t)$. We still need to show that $y_{1,2}(t)=y_{1}(t)$ and $y_{2,2}(t)=y_{2}(t)$ solve equation 16. Differentiation of (19) with respect to $t$ gives

$$
2 y_{2}^{\prime}(t)=-y_{2}^{\prime}(t) \frac{\left[1-F\left(y_{2}(t)\right)\right] f^{\prime}\left(y_{2}(t)\right)}{\left[f\left(y_{2}(t)\right)\right]^{2}}-\lambda \frac{\left[1-F\left(y_{2}(t)\right)\right]^{2}}{f\left(y_{2}(t)\right)}-R^{\prime}(1, t) .
$$

Plugging this equality into (16), we have to show that

$$
-\left(q_{2}-q_{1}\right) \lambda\left[1-F\left(y_{1,2}(t)\right)\right]\left(y_{1,2}(t)-R(1, t)\right)-\left(p_{2}-p_{1}\right) R^{\prime}(1, t)=0 .
$$

For $y_{1,2}(t)=y_{1}(t)$, this equality holds by $(20)$.

II. We now consider the case with $q_{1}=q_{2}=q$. Since $R(q, t)=q R(1, t)$, Proposition 7 implies that we can rewrite the expected revenue as

$$
q \int_{0}^{T}\left(y_{2,2}(t)+R(1, t)\right) \lambda\left(1-F\left(y_{2,2}(t)\right)\right) e^{-\int_{0}^{t} \lambda\left(1-F\left(y_{2,2}(s)\right)\right) d s} d t .
$$

The proof that the revenue maximizing cutoff curves are given by $y_{1}(t)$ and $y_{2}(t)$ is analogous to the above case, and we omit it here.

Proposition 7 implies then that

$$
R(\{1,1\}, t)=\int_{t}^{T}\left(y_{2}(s)+R(1, s)\right) \lambda\left(1-F\left(y_{2}(s)\right)\right) e^{-\int_{t}^{s} \lambda\left(1-F\left(y_{2}(z)\right)\right) d z} d s .
$$

Differentiation with respect to $t$ yields

$$
R^{\prime}(\{1,1\}, t)=\lambda\left(1-F\left(y_{2}(t)\right)\right)\left(R(\{1,1\}, t)-y_{2}(t)-R(1, t)\right) .
$$

Recall that $y_{2}(t)$ solves

$$
y_{2}(t)+R(1, t)=\frac{1-F\left(y_{2}(t)\right)}{f\left(y_{2}(t)\right)}+\lambda \int_{t}^{T} \frac{\left[1-F\left(y_{2}(s)\right)\right]^{2}}{f\left(y_{2}(s)\right)} d s
$$

Using equation (22), it is easy to verify that $R(\{1,1\}, t)$ given by equation (15) satisfies differential equation $(21)$ with the boundary condition $R(\{1,1\}, T)=0$.

To complete the proof of the Proposition we have to show that the resulting cutoffs are implementable, i.e. $y_{2}(t) \leq y_{1}(t)$ for any $t \leq T$. Noe that (18) and (19) imply that $y_{1}(t)$ is the solution to $y(t)=H(y(t))$ while $y_{2}(t)$ is the solution to $y(t)=G(y(t))$. Since $G(y) \leq H(y)$ holds for any $y$, the result follows.

For the Proof of Proposition 3, we need the following result about welfare-maximization: 
Proposition 8 (Albright, [1]) There exist $k_{t}$ unique functions $y_{k_{t}}(t) \leq y_{n-1}(t) \ldots \leq y_{1}(t)$, $\forall t$, which do not depend on the q's such that:

1. If an agent with type $x$ arrives at a time $t$, it is optimal to assign to that agent the $j$ 'th highest element of $\Pi_{t}$ if $x \in\left(y_{j}(t), y_{j-1}(t)\right]$, where $y_{0}=\infty$, and not to assign any object if $x<y_{k_{t}}(t)$.

2. For each $l$, the function $y_{l}(t)$ satisfies :

(a) $\frac{d\left[y_{l}(t)\right]}{d t}=-\lambda \int_{y_{l}}^{y_{l-1}}(1-F(x)) d x \leq 0$.

(b) $y_{j}(T)=y_{i}(T)=\inf \{x \mid f(x)>0\}$ for any $i, j \in\left\{1, \ldots, k_{T}\right\}$

Proof of Proposition 3. We start with the proof for $i=1$. From Proposition 2 we know that

$$
y_{1}^{o}(t)=\frac{1-F\left(y_{1}^{o}(t)\right)}{f\left(y_{1}^{o}(t)\right)}+\lambda \int_{t}^{T} \frac{\left[1-F\left(y_{1}^{o}(s)\right)\right]^{2}}{f\left(y_{1}^{o}(s)\right)} d s
$$

while from Proposition 8 we know that

$$
-y_{1}^{e \prime}(s)=\lambda \int_{y_{1}^{e}(s)}^{\infty}(1-F(x)) d x
$$

and that $y_{1}^{e}(T)=0$. Integrating both sides of the above differential equation between $t$ and $T$ and using the boundary condition, yields:

$$
y_{1}^{e}(t)=\lambda \int_{t}^{T}\left[\int_{y_{1}^{e}(s)}^{\infty}(1-F(x)) d x\right] d s .
$$

First, we will argue that

$$
\frac{1-F\left(y_{1}^{o}(t)\right)}{f\left(y_{1}^{o}(t)\right)}>0 \text { for any } t \in[0, T] .
$$

Assume that there exists $t^{*}$ such that

$$
\frac{1-F\left(y_{1}^{o}\left(t^{*}\right)\right)}{f\left(y_{1}^{o}\left(t^{*}\right)\right)}=0
$$

Then, since $f(x)<\infty$ for any $x,(24)$ implies that $F\left(y_{1}^{o}\left(t^{*}\right)\right)=1$. That is, the probability that an agent who arrives at time $t$ has a type above $y_{1}^{o}\left(t^{*}\right)$ is zero. By Proposition 2 , we can assume that $p=1$. This yields

$$
0<R\left(1, t^{*}\right)<y_{1}^{o}\left(t^{*}\right)
$$

Consider then decreasing $y_{1}^{o}\left(t^{*}\right)$ to $R\left(1, t^{*}\right)+\epsilon$ where $y_{1}^{o}\left(t^{*}\right)-R\left(1, t^{*}\right)>\epsilon>0$ (inequality (25) implies that such $\epsilon$ exists). This change matters only if at $t^{*}$ some agent arrives. But, in this case the proposed change increases the revenue, since the object can be sold to that agent at the price $R\left(1, t^{*}\right)+\epsilon$, while prior to the change the probability of a sale was zero. This yields a contradiction that $y_{1}^{o}\left(t^{*}\right)$ was chosen optimally.

In order to complete the proof for the one object case, it is enough (given inequality 23) to show that

$$
\forall y, \frac{(1-F(y))^{2}}{f(y)} \geq \int_{y}^{\infty}(1-F(x)) d x
$$


This follows from

$$
\int_{y}^{\infty}(1-F(x)) d x=\int_{y}^{\infty} \frac{1-F(x)}{f(x)} f(x) d x \leq \frac{1-F(y)}{f(y)}(1-F(y))
$$

where the last inequality follows by the IFR assumption.

We now proceed to the proof for two objects. After plugging in the expression for $R(1, t)$, we know from Proposition 2 that $y_{2}^{o}(t)$ solves

$$
y_{2}^{o}(t)=\frac{1-F\left(y_{2}^{o}(t)\right)}{f\left(y_{2}^{o}(t)\right)}+\lambda \int_{t}^{T}\left[\frac{\left[1-F\left(y_{2}^{o}(s)\right)\right]^{2}}{f\left(y_{2}^{o}(s)\right)}-\frac{\left[1-F\left(y_{1}^{o}(s)\right)\right]^{2}}{f\left(y_{1}^{o}(s)\right)}\right] d s
$$

By Proposition 8 we know that

$$
-y_{2}^{e \prime}(s)=\lambda \int_{y_{2}^{e}(s)}^{y_{1}^{e}(s)}(1-F(x)) d x
$$

Integrating again both sides between $t$ and $T$ yields

$$
y_{2}^{e}(t)=\lambda \int_{t}^{T}\left[\int_{y_{2}^{e}(s)}^{y_{1}^{e}(s)}(1-F(x)) d x\right] d s
$$

By Proposition 8 we also know that $y_{2}^{e}(t)<y_{1}^{e}(t)$. Together with the result for the one object case (see proof above) we obtain that $y_{2}^{e}(t)<y_{1}^{o}(t)$.

Let $y_{2}^{e}(t)$ be the solution to

$$
y(t)=H(y(t))
$$

and let $y_{2}^{o}(t)$ be the solution to

$$
y(t)=G(y(t))
$$

We are now going to show that $G(y(t))>H(y(t))$ for any $y(t)<y_{1}^{o}(t)$. Together with $y_{2}^{e}(t)<y_{1}^{o}(t)$, this will complete the proof. Note that

$$
\begin{aligned}
H(y(t)) & =\int_{t}^{T}\left[\int_{y(s)}^{y_{1}^{e}(s)}(1-F(x)) d x\right] d s \\
& =\int_{t}^{T}\left[\int_{y(s)}^{y_{1}^{e}(s)} \frac{1-F(x)}{f(x)} f(x) d x\right] d s \\
& \leq \int_{t}^{T} \frac{1-F(y(s))}{f(y(s))}\left([1-F(y(s))]-\left[1-F\left(y_{1}^{e}(s)\right)\right]\right) d s \\
& =\int_{t}^{T}\left(\frac{(1-F(y(s)))^{2}}{f(y(s))}-\frac{(1-F(y(s)))\left(1-F\left(y_{1}^{e}(s)\right)\right)}{f(y(s))}\right) d s \\
& \leq \int_{t}^{T}\left(\frac{(1-F(y(s)))^{2}}{f(y(s))}-\frac{\left(1-F\left(y_{1}^{o}(s)\right)\right)^{2}}{f\left(y_{1}^{o}(s)\right)}\right) d s<G(y(t))
\end{aligned}
$$

The third line follows from IFR assumption, and the fourth line follows from IFR, from the assumption $y(t)<y_{1}^{o}(t)$ and from $y_{1}^{e}(s)<y_{1}^{o}(t)$. The last inequality follows by the same argument as in the one object case since $\frac{1-F\left(y_{2}^{o}(t)\right)}{f\left(y_{2}^{o}(t)\right)}>0$. In addition, note that the IFR assumption implies that $G$ is a decreasing function. 
Assume now, by contradiction, that there exists some $t$ such that $y_{2}^{e}(t)>y_{2}^{o}(t)$. Then

$$
y_{2}^{e}(t)=H\left(y_{2}^{e}(t)\right)<G\left(y_{2}^{e}(t)\right)<G\left(y_{2}^{o}(t)\right)=y_{2}^{o}(t),
$$

where the first inequality follows from (26), while monotonicity of $G$ implies the second inequality. Therefore, we got that $y_{2}^{e}(t)<y_{2}^{o}(t)$, which is a contradiction. The proof for $n>2$ follows analogously.

Proof of Proposition 4. We start by showing that the expected revenue from the optimal mechanism is linear in the $q$ 's. That is, if at time $t \leq T$ the set $\Pi_{t}$ of the object is still available, then the expected revenue is given by

$$
R\left(\Pi_{t}, t\right)=\sum_{i=1}^{k_{t}} q_{(i)}\left(y_{i}(t)-\frac{1-F\left(y_{i}(t)\right)}{f\left(y_{i}(t)\right)}\right) .
$$

Our first step toward the above mentioned result consists of showing, by induction on the number of objects, that there exist factors that depend on time but not on the qualities, $a_{1}(t), \ldots, a_{k_{t}}(t)$, such that

$$
R\left(\Pi_{t}, t\right)=\sum_{i=1}^{k_{t}} q_{(i)} a_{i}(t)
$$

When only one object of quality $q$ is available, the price for the object is given by $q y_{1}(t)$ and from Claim 1 it follows that the expected revenue is given by

$$
R(q, t)=q \lambda \int_{t}^{T} \frac{\left[1-F\left(y_{1}(s)\right)\right]^{2}}{f\left(y_{1}(s)\right)} d s
$$

Assume then that if at time $t$ there are $k$ objects with qualities $\Pi_{t}=\left(q_{1}, q_{2}, \ldots q_{k}\right)$, the expected revenue is given by

$$
R\left(\Pi_{t}, t\right)=\sum_{i=1}^{k_{t}} q_{(i)} a_{i} .
$$

and consider now a situation with $k+1$ objects at time $t$. Recall that

$$
R\left(\Pi_{t}, t\right)=\sum_{i=1}^{k_{t}} \int_{t}^{T} P_{s}^{i}\left(\Pi_{t}\right) h_{i, \Pi_{t}}(s) d s+\sum_{i=1}^{k_{t}} \int_{t}^{T} R\left(\Pi_{t} \backslash\left\{q_{(i)}\right\}, s\right) h_{i, \Pi_{t}}(s) d s .
$$

The second element of the sum is linear in qualities by the induction argument, while the first element is linear by the definition of $P_{s}^{i}\left(\Pi_{t}\right)$ (see equation $(2)$ ).

To see that $a_{i}(t)=y_{i}(t)-\frac{1-F\left(y_{i}(t)\right)}{f\left(y_{i}(t)\right)}$, note that, since $a_{i}(t)$ does not depend on $\Pi_{t}$, equations (27) and (4) imply that

$$
a_{i}(t)=R\left(\mathbf{1}_{i}, t\right)-R\left(\mathbf{1}_{i-1}, t\right)=y_{i}(t)-\frac{1-F\left(y_{i}(t)\right)}{f\left(y_{i}(t)\right)}
$$

The above argument shows that, at time $t=0$, the marginal returns to qualities are ordered, with the $i$-highest produced quality having also the $i$-highest marginal return $y_{i}(0)-\frac{1-F\left(y_{i}(0)\right)}{f\left(y_{i}(0)\right)}$. By the convexity of $\phi$, the $i$-highest quality will have the $i$-highest marginal cost as well, and the characterization of the optimal produced qualities follows. As soon as the marginal return drops below $\phi^{\prime}(0)$, the cost of producing another object with a positive quality cannot be recovered, and this determines the optimal number of produced objects. 
Proof of Proposition 5. Denote by $y=\left\{y_{i}\right\}_{i=1}^{n_{1}+n_{2}}$ the optimal policy given the inventory in period $t=0$. Our previous results imply that

$$
\begin{aligned}
P_{T}^{\left(l_{1}+l_{2}\right)}\left(\Pi_{T}\right) & =s y_{l_{1}+l_{2}}(T) ; \quad P_{0}^{\left(n_{1}+n_{2}\right)}\left(\Pi_{0}\right)=s y_{n_{1}+n_{2}}(0) \\
P_{T}^{\left(l_{1}\right)}\left(\Pi_{T}\right) & =s y_{l_{1}+l_{2}}(T)+(q-s) y_{l_{1}}(T) ; \quad P_{0}^{\left(n_{1}\right)}\left(\Pi_{0}\right)=s y_{n_{1}+n_{2}}(0)+(q-s) y_{n_{1}}(0)
\end{aligned}
$$

We obtain the following chain:

$$
\begin{aligned}
\frac{P_{0}^{\left(n_{1}\right)}\left(\Pi_{0}\right)-P_{T}^{\left(l_{1}\right)}\left(\Pi_{T}\right)}{P_{0}^{\left(n_{1}\right)}\left(\Pi_{0}\right)} & \geq \frac{P_{0}^{\left(n_{1}+n_{2}\right)}\left(\Pi_{0}\right)-P_{T}^{\left(l_{1}\right)}\left(\Pi_{T}\right)}{P_{0}^{\left(n_{1}+n_{2}\right)}\left(\Pi_{0}\right)} \Leftrightarrow \\
\frac{P_{T}^{\left(l_{1}\right)}\left(\Pi_{T}\right)}{P_{0}^{\left(n_{1}\right)}\left(\Pi_{0}\right)} & \leq \frac{P_{T}^{\left(l_{1}\right)}\left(\Pi_{T}\right)}{P_{0}^{\left(n_{1}+n_{2}\right)}\left(\Pi_{0}\right)} \Leftrightarrow \\
\frac{s y_{l_{1}+l_{2}}(T)+(q-s) y_{l_{1}}(T)}{s y_{n_{1}+n_{2}}(0)+(q-s) y_{n_{1}}(0)} & \leq \frac{s y_{l_{1}+l_{2}}(T)}{s y_{n_{1}+n_{2}}(0)} \Leftrightarrow \\
\frac{q y_{l_{1}+l_{2}}(T)}{s y_{n_{1}+n_{2}}(0)+(q-s) y_{n_{1}}(0)} & \leq \frac{y_{l_{1}+l_{2}}(T)}{y_{n_{1}+n_{2}}(0)} \Leftrightarrow \\
\frac{1}{s y_{n_{1}+n_{2}}(0)+(q-s) y_{n_{1}}(0)} & \leq \frac{1}{y_{n_{1}+n_{2}}(0)} \Leftrightarrow \\
q y_{n_{1}+n_{2}}(0) & \leq s y_{n_{1}+n_{2}}(0)+(q-s) y_{n_{1}}(0) \Leftrightarrow \\
(q-s) y_{n_{1}+n_{2}}(0) & \leq(q-s) y_{n_{1}}(0) \Leftrightarrow y_{n_{1}+n_{2}}(0) \leq y_{n_{1}}(0)
\end{aligned}
$$

The third line uses Proposition 1 and Remark 2 . The fourth line uses the fact that for all $1 \leq i, j \leq n_{1}+n_{2}, y_{i}(T)=y_{j}(T)$ which is a consequence of equation 3 . The last inequality follows from Proposition 1.

Proof of Proposition 6. Assume that the inventory at time $t$ is $\Pi_{t}$, and consider two different qualities $q_{\left(j_{1}\right)}$ and $q_{\left(j_{2}\right)}$ such that $q_{\left(j_{1}\right)}>q_{\left(j_{2}\right)}$. Recall that the prices associated with each quality satisfy

$$
P_{t}^{\left(j_{l}\right)}\left(\Pi_{t}\right)=\sum_{i=j_{l}}^{k_{t}}\left(q_{(i)}-q_{(i+1)}\right) y_{i, \Pi_{t}}(t), l=1,2
$$

where $y$ denotes the (quality independent) revenue maximizing allocation policy. Thus,

$$
P_{t}^{\left(j_{1}\right)}\left(\Pi_{t}\right)=P_{t}^{\left(j_{2}\right)}\left(\Pi_{t}\right)+\sum_{i=j_{1}}^{j_{2}-1}\left(q_{(i)}-q_{(i+1)}\right) y_{i, \Pi_{t}}(t)
$$

which implies

$$
\frac{d P_{t}^{\left(j_{1}\right)}\left(\Pi_{t}\right)}{d t}=\frac{d P_{t}^{\left(j_{2}\right)}\left(\Pi_{t}\right)}{d t}+\frac{d\left[\sum_{i=j_{1}}^{j_{2}-1}\left(q_{(i)}-q_{(i+1)}\right) y_{i, \Pi_{t}}(t)\right]}{d t}
$$

Lemma 1 (proven below) yields that

$$
\frac{d y_{i, \Pi_{t}}(t)}{d t} \leq 0, \forall i
$$

which implies

$$
\frac{d P_{t}^{\left(j_{1}\right)}\left(\Pi_{t}\right)}{d t} \leq \frac{d P_{t}^{\left(j_{2}\right)}\left(\Pi_{t}\right)}{d t}
$$


Since these last derivatives are negative (which is implied again by Lemma 1 ) the result follows ${ }^{20}$.

Lemma 1 In the revenue maximizing mechanism, the cutoffs determining the allocation policy are decreasing with time. That is

$$
y_{i}^{\prime}(t) \leq 0 \text { for } i \in\left\{1, \ldots, k_{t}\right\} \text { and } t \in[0, T) .
$$

Proof. We prove the result by induction on the number of the available objects $k_{t}$. If $k_{t}=1$, differentiating (11) w.r.t. $t$ gives

$$
y_{1}^{\prime}(t) \frac{\partial}{\partial y_{1}(t)}\left(y_{1}(t)-\frac{1-F\left(y_{1}(t)\right)}{f\left(y_{1}(t)\right)}\right)=-\lambda \frac{\left[1-F\left(y_{1}(t)\right)\right]^{2}}{f\left(y_{1}(t)\right)} .
$$

The result follows then since the r.h.s. is negative, and since the virtual valuation is assumed to be increasing. Assume then that the statement holds for $k_{t}=l$, and consider the case where $k_{t}=l+1$. The properties of the revenue maximizing mechanism imply that $l$ highest curves $y_{i}(t)$ coincide with those relevant for the case where $k_{t}=l$. Thus $y_{i}^{\prime}(t) \leq 0$ for $i \in\{1, \ldots, l\}$. Differentiating w.r.t. $t$ the expression (3) for $y_{l+1}(t)$ gives

$$
y_{l+1}^{\prime}(t) \frac{\partial}{\partial y_{l+1}(t)}\left(y_{l+1}(t)-\frac{1-F\left(y_{l+1}(t)\right)}{f\left(y_{l+1}(t)\right)}\right)=\lambda\left[\frac{\left[1-F\left(y_{l}(t)\right)\right]^{2}}{f\left(y_{l}(t)\right)}-\frac{\left[1-F\left(y_{l+1}(t)\right)\right]^{2}}{f\left(y_{l+1}(t)\right)}\right] .
$$

Proposition 1 implies that $y_{l+1}(t) \leq y_{l}(t)$ for any $t \in[0, T)$. There are two cases:

1. There exists $t \in[0, T)$ such that $y_{l+1}(t)=y_{l}(t)$. Then, (28) implies that $y_{l+1}^{\prime}(t)=0$.

2. For any $t \in[0, T)$ we have $y_{l+1}(t)<y_{l}(t)$. Notice that the function $\frac{[1-F(y)]^{2}}{f(y)}$ is decreasing if and only if the virtual valuation is increasing. Therefore, an increasing virtual valuation implies that the r.h.s. of $(28)$ is negative and that $\frac{\partial}{\partial y_{l+1}(t)}\left(y_{l+1}(t)-\frac{1-F\left(y_{l+1}(t)\right)}{f\left(y_{l+1}(t)\right)}\right)>$ 0 . This yields $y_{l+1}^{\prime}(t)<0$, and completes the proof.

\footnotetext{
${ }^{20}$ The same argument also shows that the difference between the two prices is monotonically decreasing.
} 